\title{
A valid method of gas foil bearing parameter estimation: A model anchored on experimental data
}

Proc IMechE Part C:

$J$ Mechanical Engineering Science 2018, Vol. 232(24) 45I0-4527 (C) IMechE 2016

Article reuse guidelines:

sagepub.com/journals-permissions DOI: 10.1 177/0954406216667966 journals.sagepub.com/home/pic

@SAGE

\author{
Robert Hoffmann, Oliver Munz, Tomasz Pronobis, \\ Enrico Barth and Robert Liebich
}

\begin{abstract}
Gas foil bearings are a smart green technology and suitable for the next generation of small turbo machinery e.g. turbochargers, micro gas turbines, range extenders and compressors of fuel cells. A combination of low power loss, high speed operation and the omission of an oil system are the major advantages. To enable access to this technology, it is essential to evaluate critical speeds and onset speeds of subharmonic vibration of the rotor system in the first design stage. Hence, robust and valid models are necessary, which correctly describe the fluid structure interaction between the lubrication film and the elastic bearing structure. In the past three decades several experimental and numerical investigations of bearing parameters have been published. But the number of sophisticated models is small and there is still a lack of validation towards experimental works. To make it easy for designers dealing with this issue, the bearing parameters are often linearised about certain operating points. In this paper a method for calculating linearised bearing parameters (stiffness and damping) of gas foil bearing is presented. Experimental data are used for validation of the model. The linearised stiffness and damping values are calculated using a perturbation method. The pressure field is coupled with a two-dimensional plate model, while the non-linear bump structure is simplified by a link-spring model. It includes Coulomb friction effects inside the elastic corrugated structure and captures the interaction between the single bumps. For solving the separated perturbed Reynolds equation a static stiffness is used for the 0 . order equation (stationary case) and a dynamic stiffness is applied for I. order equation (dynamic case). Therefore, an additional dynamic structural model is applied to calculate the dynamic stiffness. The results depend on the load level and friction state of each bump. Different case studies including the impact of clearance, frictional contacts and the comparison of a linear and non-linear structure are carried out for infinitesimal perturbations. The results show, that the linear structure underestimates main and cross-coupling effects. The impact of the clearance is notable, while the impact of the overall frictional contacts is small due to relatively small loadings. The infinitely small perturbation model is adapted to the experimental setup by using a superposition of two resulting bearing parameters identifications of two total loadings including shaker forces. Due to this adaptation a good correlation with the experimental results of the bearing parameters is achieved.
\end{abstract}

\section{Keywords}

Gas foil bearing, parameter identification, non-linear structure, friction

Date received: 30 March 2016; accepted: 9 August 2016

\section{Introduction}

In the last three decades gas foil bearings (GFBs) have successfully been introduced into turbo machinery. Bump type GFBs comprise an elastic bump and top foil. The foils are fixed with the bearing sleeve e.g. by spot welds. Fluid dynamic pressure deforms the elastic structure and generates additional damping due to frictional contacts. Key issues are poor overall damping level, low load capacity due to the low viscosity of the air film and the lack of validated numerical models. In the past, 'trial and error' methods have been applied to improve poor damping and load capacities. In particular, the poor damping level cannot prevent sub-synchronous vibrations characterised by limit cycles. These vibrations occur at

Chair of Engineering Design and Product Reliability, Berlin Institute of Technology, Germany

\section{Corresponding author:}

Robert Hoffmann, Berlin Institute of Technology (TU Berlin), Str. d. I7. Juni 135, 10623 Berlin, Germany.

Email: robert.hoffmann@posteo.de 
higher rotor speeds, due to self-excitations, ${ }^{1}$ and poor balanced systems. $^{2-7}$ The use of structural modifications on the elastic structure can avoid or decrease these vibrations. ${ }^{2-4}$

In Hoffmann et al. ${ }^{1}$ the source of the sub-synchronous vibrations is analysed. A thesis is stated, that two non-linearities are responsible: The fluid film and the non-linear elastic structure. Well-balanced rotor systems will be self-excited by the fluid film. Considering the self-excitation, a Hopf-Bifurcation can describe the onset speed of sub-synchronous vibrations $\Omega_{0}$. The eigenvalue $\lambda_{i}=\delta_{i} \pm \mathrm{j} \omega_{i}^{*}$ of the Jacobi matrix of an autonomous system, where the damping ratio $\delta_{i}=0$ of the mode $i$ eigenvalue becomes zero, is related on a Hopf-Bifurcation. ${ }^{8,9}$ As Hoffmann et al. show, ${ }^{1}$ the onset speed $\Omega_{0}$ can be estimated by using an approximation based on linearised bearing parameters. This method agrees well with the results of a time domain analysis. In detail, linearised bearing parameters (stiffness $\mathbf{K}(\Omega, \omega)$ and damping $\mathbf{C}(\Omega, \omega)$ ) are applied in a linear eigenvalue problem by solving the homogeneous linear ODE-system, equation (1)

$$
\mathbf{M} \ddot{\mathbf{u}}+(\mathbf{C}(\Omega, \omega)-\mathbf{G}(\Omega)) \dot{\mathbf{u}}+\mathbf{K}(\Omega, \omega) \mathbf{u}=\mathbf{0}
$$

where the global matrices for mass $\mathbf{M}$, damping $\mathbf{C}(\Omega, \omega)$ and stiffness $\mathbf{K}(\Omega, \omega)$ are based on the given rotor structure. Gyroscopic effects are captured by $\mathbf{G}(\Omega)$. Note that the system depends on the rotation speed $\Omega$ and due to the compressible gas of the bearings on the excitation frequency $\omega$. Therefore, the eigenvalues $\lambda_{i}(\Omega, \omega)=\delta_{i}(\Omega, \omega) \pm \mathrm{j} \omega_{i}^{*}(\Omega, \omega)$ are a function of the rotational speed $\Omega$ and the excitation frequency $\omega$. The intersection, where the real part of an eigenvalue becomes zero $\left(\delta_{i}\left(\Omega_{0}, \omega\right)=0\right)$ and the imaginary part is equal to the excitation frequency $\left(\mathrm{j} \omega_{i}^{*}\left(\Omega_{0}, \omega\right)=\omega\right)$, states the point of a self-excitation (Figure 1). Hence, a correct and robust estimation of critical speed $\Omega_{0}$ is significant for the whole design process and is essential to avoid additional costs.

However, to keep the estimation error as small as possible a valid method for a parameter identification

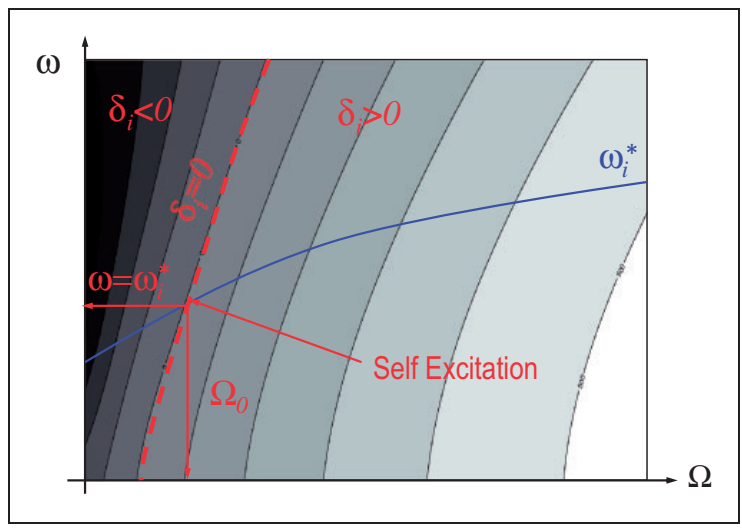

Figure I. Evaluation of the self excitation: $\delta_{i}$ vs. $\Omega$ vs. $\omega_{i}^{*}$. has to be applied. In the past, several numerical models to calculate the linearised bearing parameters have been published. The parameters can be calculated by using the infinitely small perturbation method of Lund. ${ }^{10}$ Where the numerical parameter identifications consider simple linear structural models $^{11-18}$ or using complex non-linear structural models, which take bump interactions and frictional contacts into account. ${ }^{19-25}$ However, no detailed validation with experimental linearised bearing parameters is shown. Only the study of Larsen et al. ${ }^{19}$ includes a validation with experimental data of a second generation GFB, but it is limited to one loading case $\left(W_{x}=200 \mathrm{~N}\right.$ at $\left.20,000 \mathrm{r} / \mathrm{min}\right)$ and only sub-synchronous excitation is shown. One important outcome of this study is that the authors distinguish between a static and dynamic stiffness, which improves the agreement to the measured parameters. Unfortunately, only a small number of published experimental parameter identification data of GFBs is published. ${ }^{20-30}$ However, Rudloff et. $\mathrm{al}^{28}$ deliver the widest field of experimental data for first generation bump type GFB. The study includes several load levels $(10-50 \mathrm{~N})$, rotor speeds $(15,600-35,400 \mathrm{r} / \mathrm{min})$ and excitation frequencies $(100-600 \mathrm{~Hz})$.

The purpose of this paper is to deliver a valid method for a gas foil bearing parameter estimation. To achieve realistic results the fluid film is coupled with a non-linear structure model for the static and dynamic cases. First, the dynamic structure model is validated with experimental data from Larsen et al. ${ }^{31}$ In the second part of the paper, the linearisation model is validated with Rudloff's experiments. ${ }^{28}$ The present paper shows a theory for an improvement of the correlation between linearised simulations and experimental parameter identification. The theory is based on adapting higher dynamic shaker forces, if they are in the same range of static loadings. In addition, the impact of frictional contacts and nominal clearance is analysed, because these parameters are affected by uncertainties during the design process.

\section{Theoretical model}

\section{Fluid film}

Figure 2 shows a compliant structure of a GFB in a inertial coordinate system $(x, y$ and $\theta$ ). A turning journal with an angular speed $\Omega$ and a centre displacement $e_{x}$ and $e_{y}$ generates a forced slip stream with a film thickness of $h(z, \theta)$. It results in a dynamic pressure field $p(z, \theta)$, which produces a reacting force $\mathbf{f}_{B}$. An equilibrium condition is reached if the sum of the loading vector $\mathbf{w}=\left\{W_{x}, W_{y}\right\}^{T}$ and the reacting force vector $\mathbf{f}_{B}$ is zero. An integration of the pressure field along the axial and circumferential directions yields a reacting force vector $\mathbf{f}_{B}$, equation (2). It acts under the attitude angle $\beta$. Note that the axial direction $z$ is 


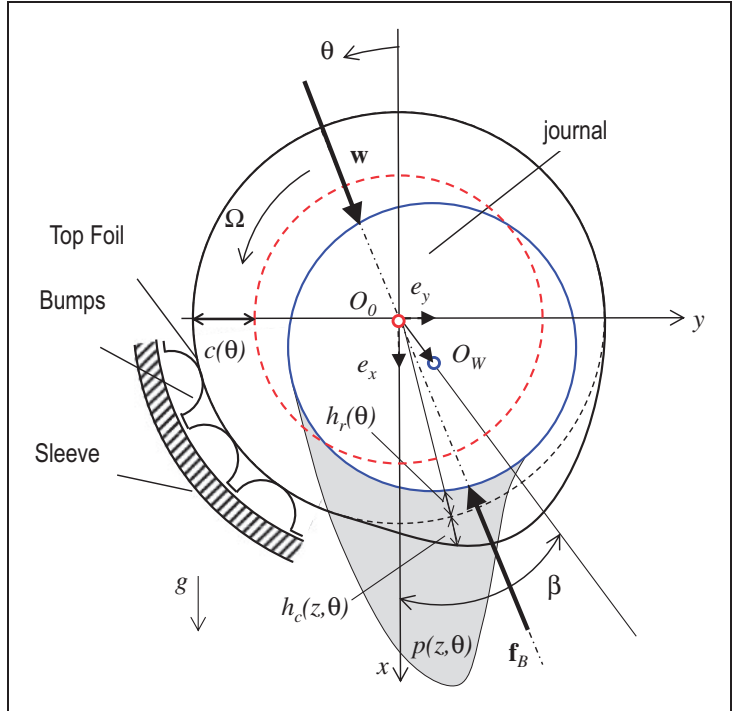

Figure 2. GFB with a dynamic pressure field.

related to the bearing length $l$.

$$
\mathbf{f}_{B}=R \int_{0}^{l} \int_{0}^{2 \pi}\left(p(z, \theta)-p_{a}\right)\left\{\begin{array}{c}
\cos \theta \\
\sin \theta
\end{array}\right\} \mathrm{d} z \mathrm{~d} \theta
$$

Due to the pressure field, an elastic deformation of the foil structure is given by $h_{c}(z, \theta)$ and is calculated by a structural model as shown below. Equation (3) describes a perfectly aligned journal, expansion effects due to temperature gradients and centrifugal forces are neglected. Note that these effects should be taken into account if thin journal walls and high temperature operations are present, which is neglected in this work. The film thickness is composed of a rigid term $h_{r}(\theta)$, including the nominal clearance $c$ and the journal centre displacements, and of a compliant term $h_{c}(z, \theta)$. The clearance is a theoretical parameter, which can be estimated experimentally. Further structural modifications, e.g. shimming, may affect the clearance in a circumferential direction, ${ }^{1,2}$

$$
h(\theta, z)=\underbrace{c+e_{x} \cos (\theta)+e_{y} \sin (\theta)}_{h_{r}(\theta)}+h_{c}(z, \theta)
$$

The pressure field $p(z, \theta)$ is calculated by solving the Reynolds equation (RE) for a compressible, isothermal and isoviscous fluid (equation (4)). It links both pressure field and film thickness under the presence of journal rotation speed $U=R \Omega$. The following boundary conditions are applied: $p(z=0, \theta)=p(z=l, \theta)$ $=p_{a}$ and $p(z, \theta=0)=p(z, \theta=2 \pi)=p_{a}$

$$
\begin{gathered}
\frac{\partial}{\partial z}\left(p h^{3} \frac{\partial p}{\partial z}\right)+\frac{1}{R^{2}} \frac{\partial}{\partial \theta}\left(p h^{3} \frac{\partial p}{\partial \theta}\right) \\
\quad=\frac{\Omega \mu_{l}}{2} \frac{\partial(p h)}{\partial \theta}+12 \mu_{l} \frac{\partial(p h)}{\partial t}
\end{gathered}
$$

If the pressure falls underneath the ambient pressure condition $p(z, \theta)<p_{a}$, a top foil lift-off occurs and subambient pressures is reaching the ambient level $\left(p=p_{a}\right)$. Thus, sub-ambient pressures are set to $p=p_{a}$. Bearing parameters are calculated by using Lunds approach, ${ }^{10}$ which is based on a perturbation method. In the equilibrium state of a given static load w under steady speed condition $(\Omega=$ const.) a harmonic perturbation is superimposed with a frequency $\omega$ and infinitely small eccentricity $\left(\Delta e_{x, y} \ll c\right)$. In addition, the pressure field and the film thickness are affected by perturbations, where $i=x, y$. Substituting equation (5) into equation (4), while neglecting terms of higher order, generates zero and first order Reynolds equations, which have to be successively solved.

$$
\begin{aligned}
& e=e_{0}+\Delta e_{i} e^{\mathrm{j} \omega t} \\
& p=p_{0}+\Delta e_{i} p_{i} e^{\mathrm{j} \omega t} \\
& h=h_{0}+\Delta e_{i}\left(h_{i}+h_{c, i}\right) e^{\mathrm{j} \omega t}
\end{aligned}
$$

The perturbed pressure fields $p_{x}$ and $p_{y}$ are used to calculate the linearised stiffness and damping matrices (equation (1) and (8)). The calculation delivers complex matrix elements that are known as bearing impedance $z_{i j}=k_{i j}+\mathrm{j} \omega c_{i j}$, where $i, j=x, y$. A more detailed explanation is given by Kim and San Andrés ${ }^{11}$

$$
\begin{aligned}
& {\left[\begin{array}{ll}
z_{x x} & z_{x y} \\
z_{y x} & z_{y y}
\end{array}\right]=\underbrace{\left[\begin{array}{ll}
k_{x x} & k_{x y} \\
k_{y x} & k_{y y}
\end{array}\right]}_{\mathbf{K}(\omega)}+\mathrm{j} \omega \underbrace{\left[\begin{array}{cc}
c_{x x} & c_{x y} \\
c_{y x} & c_{y y}
\end{array}\right]}_{\mathbf{C}(\omega)}} \\
& =-R^{2} \int_{0}^{l} \int_{0}^{2 \pi}\left[\begin{array}{cc}
p_{x} \cos \theta & p_{y} \cos \theta \\
p_{x} \sin \theta & p_{y} \sin \theta
\end{array}\right] \mathrm{d} z \mathrm{~d} \theta
\end{aligned}
$$

\section{Structural model}

A detailed description of the two non-linearities caused by the fluid film and the elastic structure is necessary in order to achieve valid results; a simple as well as detailed model needs to be introduced. It includes following features and assumptions:

- top foil displacement including axial and circumferential components;

- non-linear frictional contacts between top- and bump-foil and between bump-foil and housing are taken into account by using the Coulomb model for static and dynamic analyses;

- each bump $i$ can interact with its surrounding the preceding $i-1$ and the subsequent bump $i+1$;

- the applied bump load $F_{p}$ is concentrated on the top centre of the bump; 


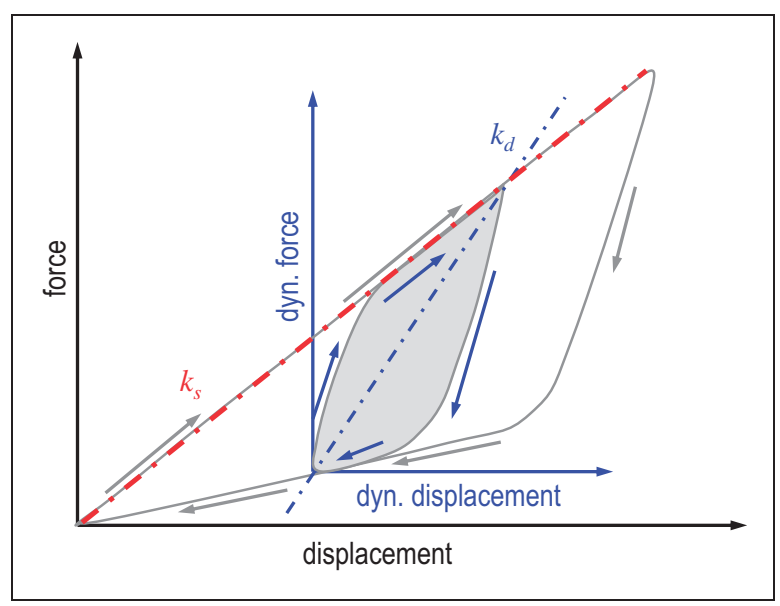

Figure 3. Schematic hysteresis according to Larsen et al. ${ }^{19}$

- bumps are reduced towards rigid segments linked by pivots, while bump interactions are transmitted by linear springs;

- bump deformation along the axial direction is assumed to be constant;

- no bump-foil separation from the housing is possible;

- all deformations are elastic.

Recently, Larsen et al. ${ }^{19}$ have shown that small perturbations around an equilibrium position result in significantly higher slopes in hysteresis (stiffer system) compared to a static load path, as shown in Figure 3. Due to the decreased slip phase the sticking phase of the hysteresis is dominating. Hence, stiffness will increase with a decreasing of the dynamic displacement amplitudes and two structural stiffness $k_{s}$ and $k_{d}$ have to be calculated, where the static stiffness $k_{s}$ is used for the zeroth order RE (static stationary case) and the dynamic stiffness $k_{d}$ is applied to solve the perturbed first order RE (dynamic case). For the two kinds of stiffness different models are applied.

However, both models are using the same finite element (FE) structure, where a realistic structure (Figure 4(a)) is reduced to an equivalent model (Figure 4(b)): The top foil is described by a thin two-dimensional (2D) plate, where three-dimensional (3D) effects due to normal tensions are neglected. These finite plate elements are linked to equivalent non-linear bump spring-damper elements, calculated by the structural models. Both the static and dynamic bump matrix $\mathbf{K}_{\text {Bump }}\left(\mathbf{f}_{p}, \mathbf{f}_{f}\right)$ is a function of the loading state of the pressure field $\mathbf{f}_{p}$ and the frictional contact forces $\mathbf{f}_{f}$. In addition, the top foil's Young-modulus $E$ is increased by a factor of 4 to obtain appropriate sagging effects between adjacent bumps. ${ }^{16}$

Static structural bump model. An equivalent link-spring model is used to describe the structural behaviour; it is

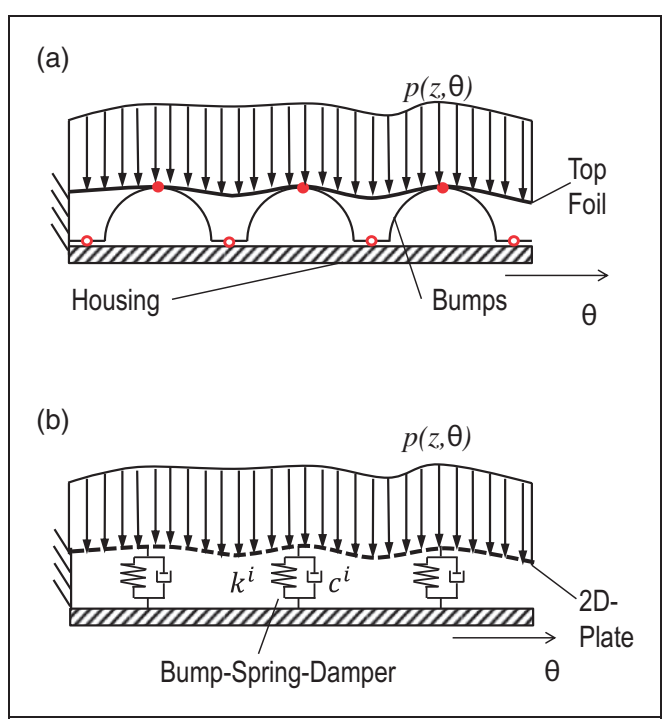

Figure 4. GFB Structure: (a) real structure and (b) model (bump spring and damper model and top foil plate model).

mainly based on the work of Feng et al. ${ }^{21}$ Figure 5(a) shows the free punch and the kinematic of the pivot.

The force equilibrium equation (9) is given for a bump segment 'I'. The contact forces between bump/ housing (index bot) and bump/top foil (index up) are included

$$
F_{\mathrm{bot}}^{i}=F_{s}^{i+1}+F_{b, x}^{i}-F_{s}^{i}-F_{b, x}^{i+1} \equiv F_{x}^{i}
$$

where beam lever forces for the $i$ and $i+1$ bump segments are given by $F_{b, x}^{i}=F_{r}^{i} / \tan \left(\alpha_{i}\right)$ and $F_{b, x}^{i+1}=$ $F_{l}^{i} / \tan \left(\alpha_{i}\right)$, as well as the interaction forces $F_{s}^{i}=2 k_{1} \Delta L^{i}$ and $F_{s}^{i+1}=2 k_{1} \Delta L^{i+1}$ due to horizontal displacement $\Delta L^{i}$ and $\Delta L^{i+1}$ of the linear spring $k_{1}$, based on the Castigliano theorem. ${ }^{21}$ The right- and leftward forces include the friction force $F_{\text {up }}^{i}$ and the concentrated bump loading force $F_{p}^{i}$

$$
F_{r}^{i}=\frac{F_{p}^{i}}{2}-\frac{F_{\text {up }}^{i}}{2} \tan \left(\alpha^{i}\right)
$$

and

$$
F_{l}^{i}=\frac{F_{p}^{i}}{2}+\frac{F_{\mathrm{up}}^{i}}{2} \tan \left(\alpha^{i}\right)
$$

For the static structure model the motion state is checked by the force equilibrium equation (9), where the sign-function of the Coulomb model is considered. Finally, the equivalent stiffness can be calculated by estimating the motion states and can be introduced into the FE-model structure. ${ }^{23}$ If both segments are in a stick condition the kinematic of the bump is changed and delivers a higher stiffness (Figure 5(c)).

Dynamic structural bump model. To calculate the dynamic hysteresis of a bump structure, equation (9) has to be solved in the time domain, while varying 


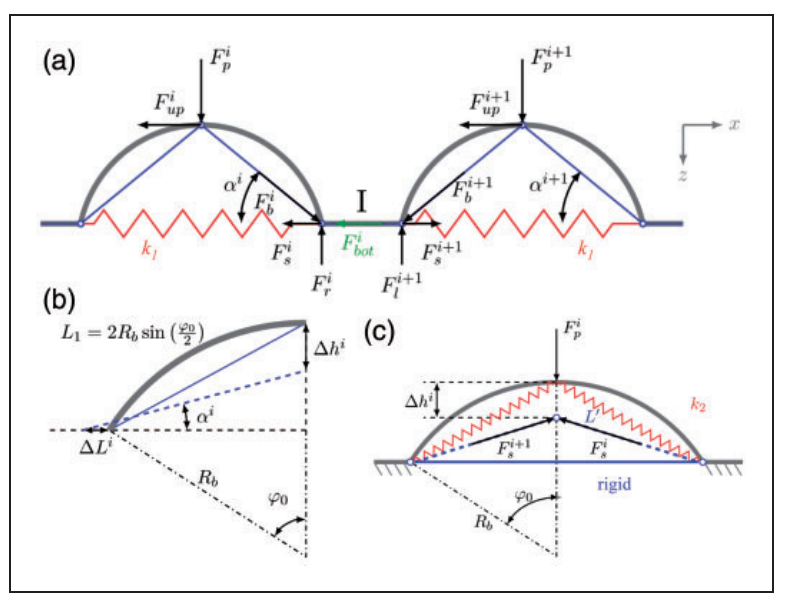

Figure 5. Linked-spring-model: (a) Free punch of a single interacting bump segment I. (b) Kinematic of a rigid bump lever and (c) stick-stick condition.

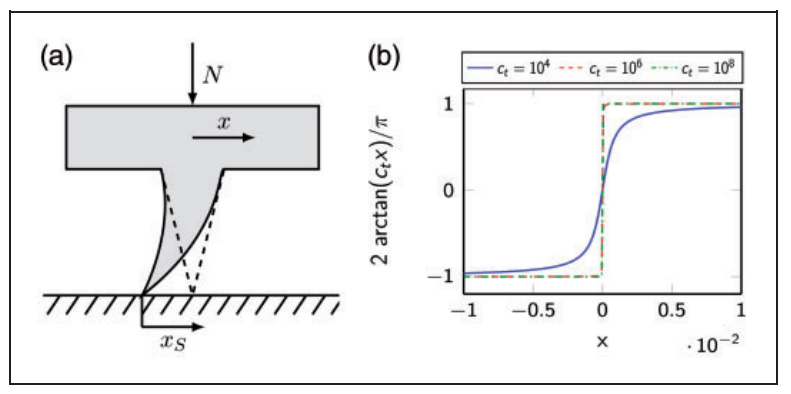

Figure 6. (a) Schematic brush model. (b) Impact of the Form parameter $c_{t}$ on the sign-approximation.

$\Delta h$ or $F_{p}$. For higher excitation frequencies the Grosssliding regime becomes smaller. ${ }^{32}$ Hence, the Stribeck effects are reduced and the frequency has no significant impact on the structural hysteresis, which has been experimentally shown. ${ }^{33}$ Thus, the Stribeck effect is neglected and only Coulomb friction is used. Unfortunately, the Coulomb friction that considers the sign-function leads to numerical problems. The introduction of the dynamic friction model of Petrov and Ewins ${ }^{34}$ avoids that problem and a time domain analysis is possible. This approach has been successfully introduced in GFB structures already by Le Lez et al. ${ }^{35}$ The frictional forces due to acting normal force $N$ can be described by a relative deflection $\left(x-x_{s}\right)$ of a simple brush model with the stiffness $k_{t}$ (Figure 6(a))

$$
F=k_{t}\left(x-x_{s}\right) \equiv\left(\dot{x}_{S}\right) \mu N \text { sign }
$$

To overcome unsteadiness problems of the sign-function, an approximation is applied by using an arctangent function

$$
\operatorname{sign}\left(\dot{x}_{S}\right) \approx \frac{2}{\pi} \arctan \left(c_{t} \dot{x}_{S}\right)
$$

The approximation includes $c_{t}$, which is a model parameter that controls the slope of the arctangent function (Figure 6(b)). A numerical investigation with a multiple parameter variation (E-modulus, bump number, load level, and $k_{t}$-parameter) shows that applying a value of $c_{t} \geqslant 6 \times 10^{6}$ in a $k_{t}$ range of $\left[4 \times 10^{5}, 10^{6}\right] \mathrm{N} / \mathrm{m}$ the numerical results of a dynamic hysteresis become independent of the slope parameter $c_{t}{ }^{36}$ However, applying too high $c_{t}$ values increases significantly the solution time. Hence, $c_{t}=6 \times 10^{6}$ is chosen. Finally, a time deviation of equation (11) and substitution of equation (13) in equation (12) yields to equation (13)

$$
\frac{\mathrm{d} F}{\mathrm{~d} t}=k_{t}\left(\dot{x}-\frac{1}{c_{t}} \tan \left(\frac{\pi F}{2 \mu N}\right)\right)
$$

Applying this method on a GFB structure, the bump/ top foil contact (index up) and bump segment/housing contact (index bot) are given by equations (14)

$$
\begin{aligned}
& \dot{F}_{\text {up }}^{i}=k_{t}\left(\dot{x}_{\text {up }}^{i}-\frac{1}{c_{t}} \tan \left(\frac{\pi F_{\text {up }}^{i}}{2 \chi N_{\text {up }}^{i}}\right)\right) \\
& \text { and } \\
& \dot{F}_{\text {bot }}^{i}=k_{t}\left(\dot{x}_{\text {bot }}^{i}-\frac{1}{c_{t}} \tan \left(\frac{\pi F_{\text {bot }}^{i}}{2 \mu N_{\text {bot }}^{i}}\right)\right)
\end{aligned}
$$

A time deviation of the force equilibrium of a single bump segment and substituting the dynamic friction force results to

$$
\begin{aligned}
\frac{\mathrm{d}}{\mathrm{d} t}\left(\frac{F_{p}}{\tan (\alpha)}\right)_{i}= & 2 k_{1}\left(2 \dot{x}_{\text {bot }}^{i}-\dot{x}_{\text {bot }}^{i-1}-\dot{x}_{\text {bot }}^{i+1}\right) \\
& +\frac{\mathrm{d}}{\mathrm{d} t}\left(\frac{F_{p}}{\tan (\alpha)}\right)_{i+1}+2 \dot{F}_{\text {bot }}^{i}+\dot{F}_{\text {up }}^{i}+\dot{F}_{\text {up }}^{+1}
\end{aligned}
$$

Furthermore, the kinematic relationships $2 \Delta L^{i}=$ $x_{\mathrm{bot}}^{i-1}-x_{\mathrm{bot}}^{i+1}$ and $2 x_{\mathrm{up}}^{i}=x_{\mathrm{bot}}^{i}+x_{\mathrm{bot}}^{i-1}$ are used. Due to low masses of the GFB structure inertia effects are neglected. The equation is a non-linear ODE-system and can be written in a matrix form

$$
\underset{\mathrm{bot}}{\mathbf{u}}=\mathbf{A}\left(x_{\mathrm{bot}}\right)^{-1} \mathbf{f}
$$

An ODE-solver for stiff problems, Matlab's ode23s(...), ${ }^{35}$ is used for solving equation (16) in the time domain. For more details of the structural models, including a validation based on numerical results, see Hoffmann et al. ${ }^{23}$ The overall calculation process is shown in Figure 7.

\section{Validation of the dynamic structure model}

Figure 8 shows the numerical results of the presented dynamic structural model vs. experimental data from Larsen et al. ${ }^{31}$ Two bump strips with three and four 
bumps (Inconel X-750, $s_{b}=7 \mathrm{~mm}, \quad l_{b}=3.3 \mathrm{~mm}$, $l=18 \mathrm{~mm} h_{b}=0.9 \mathrm{~mm}, t_{B}=0.127 \mathrm{~mm}$ and $\mu=0.12$ ) are considered. Both bump strips have a static loading of $40 \mathrm{~N}$ and are excited by a dynamic load with $1 \mathrm{~Hz}$ excitation frequency. The dynamic displacement amplitudes $\Delta \hat{h}$ are kept constant between two and $12 \mu \mathrm{m}$. For the dynamic model optimised model parameters are set to $k_{t}=312-585 \mathrm{kN} / \mathrm{m}$ and $c_{t}=6 \cdot 10^{10}$. The high value of the model parameter $c_{t}$ is a compromise between the lowest possible impact on the numerical results and economical simulation times. Both numerical results show good agreement with the

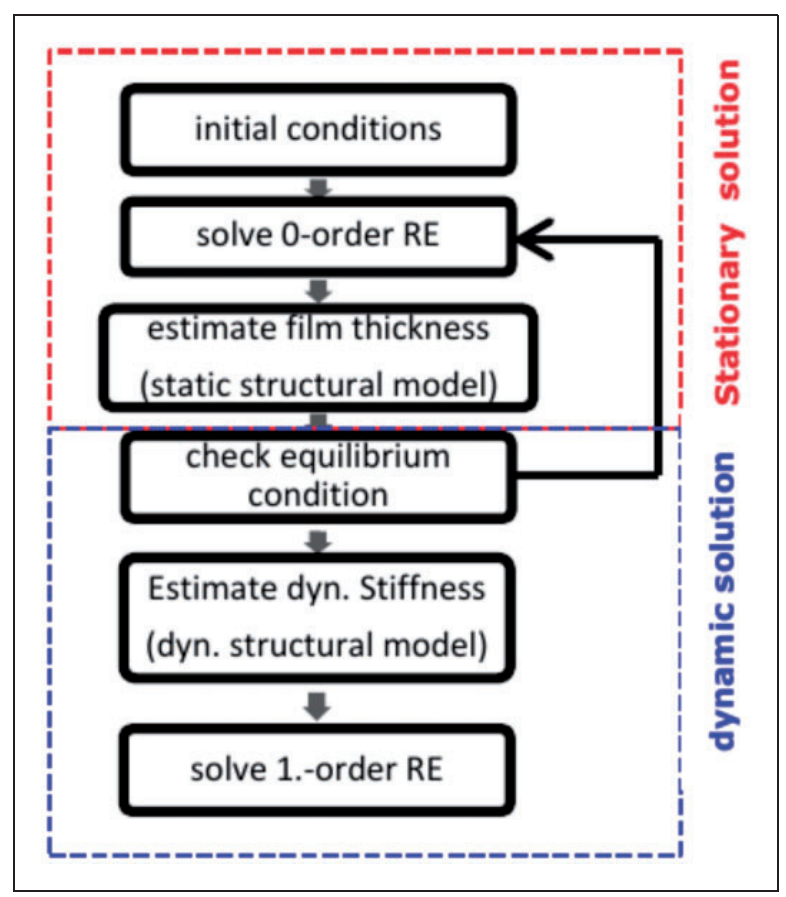

Figure 7. Model routine. experimental data. Note that increasing dynamic displacement results in smaller hysteresis slopes. The loading path in stick (s) and sliding phase (m) correlates well. However, the unloading path has smaller stick phases (s) in the simulation. Hence, the enclosed area of the calculated hysteresis is smaller than the experimental results: smaller energy is dissipated by the numerical simulation. This effect is more present for higher displacements and for the stripe with four bumps. Due to the macroscopic contact modelling by using the Petrov model changes of the contact surface characteristics, e.g. curvature effects of the bumps, are neglected. Thus, the effect is more notable for four bumps due to the higher number of contacts. Furthermore, Larsen et al. ${ }^{31}$ have a very soft structure. Higher displacement amplitudes may generate non-linear displacement characteristics and interact with the contacts. These non-linear effects are neglected in the link-spring structure.

\section{Validation of the GFB parameter identifi-cation model}

In this work the experimental results of Rudloff et al. $^{28}$ are used. The data of the first generation GFB is listed in Table 1. In general, a linearisation of bearing parameters is based on small perturbations around an equilibrium position. Applying Lunds approach $^{10}$ will deliver theoretical bearing parameters, where infinitely small perturbations are assumed. Hence, to compare measurements with simulations based on infinitely small perturbations it is necessary to achieve relatively small perturbations during experiments. As Qiu and Tieu show ${ }^{37}$ (related on an oil lubricated journal bearing parameter identification) the displacements have to be kept relatively small $(0.02 c ; 2 \%$ of the nominal clearance $c)$ to

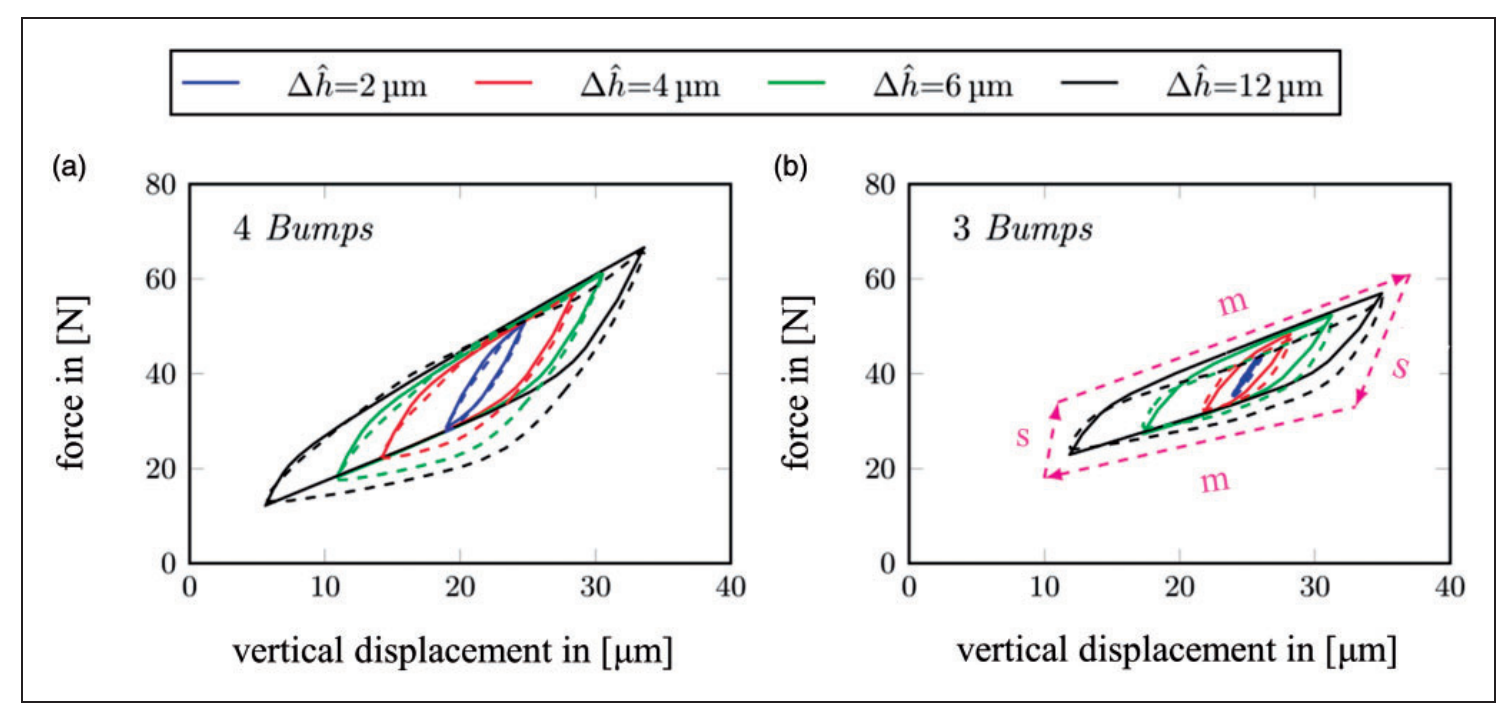

Figure 8. Numerical results (solid line) vs. experimental data from Larsen et al. ${ }^{31}$ (dashed line). Dynamic hysteresis for four bumps (a) $\left(k_{t}=585 \mathrm{kN} / \mathrm{m}\right)$ and three bumps (b) $\left(k_{t}=312 \mathrm{kN} / \mathrm{m}\right)$ under $40 \mathrm{~N}$ static loading. Dynamic displacement amplitudes $2-12 \mu \mathrm{m}$. 
Table I. Data for the first generation gas foil bearings. ${ }^{28}$

\begin{tabular}{ll}
\hline Parameter & Value \\
\hline Bearing radius $R$ & $19.050 \mathrm{~mm}$ \\
Bearing length I & $38.100 \mathrm{~mm}$ \\
Bump height $h_{b}$ & $0.5 \mathrm{~mm}$ \\
Bump thickness $t_{b}$ & $0.102 \mathrm{~mm}$ \\
Bump pitch $s_{b}$ & $4.566 \mathrm{~mm}$ \\
Bump number $N_{b}$ & 26 \\
Half bump length $I_{b}$ & $1.778 \mathrm{~mm}$ \\
Top foil thickness $t_{f}$ & $0.102 \mathrm{~mm}$ \\
Young-modulus $E$ & $2.07 \times 10^{\prime \prime} \mathrm{N} / \mathrm{m}^{2}$ \\
Poisson ratio $v$ & 0.3 \\
Friction coefficient $\mu$ & 0.1 \\
& \\
\hline
\end{tabular}

achieve acceptable results between small and infinitely small displacement theory. In addition, Lund shows that gas foil bearing parameter identification works reasonably will up to $0.4 c$ excitation displacements ${ }^{38}$ However, studies considering elastic structures have not been published yet.

Furthermore, most of the works (e.g. Rudloff et al. $^{28}$ ) use an electromagnetic shaker to excite the system. In order to achieve a quasi linear behaviour, the applied dynamic loading has to be small compared to the static bearing loading. If the dynamic forces are too high increased displacements will be generated. Hence, significant deviations between experiments and numerical simulation can occur. In this work Rudloff et al's. mean data are used. ${ }^{28}$ Unfortunately, no information on the measurement accuracy and standard deviations of the parameters are given.

\section{Linear vs. non-linear structural model}

In the first section, the experimental data were compared with the calculations based on infinitely small perturbations. In addition, a comparison between the numerical results for a non-linear bump structural model, where the static and dynamic bump stiffnesses were considered, along with a simple linear spring model for the bump structure ${ }^{11-18}$ is shown. This analysis delivers necessary information about the impact on parameter estimation by using a linear structural model. The model parameter of the non-linear structural dynamic bump model is chosen with $c_{t}=6 \cdot 10^{10}$, while $k_{t}$ has been identified by experimental structural dynamic tests of a gas foil bearing with the same

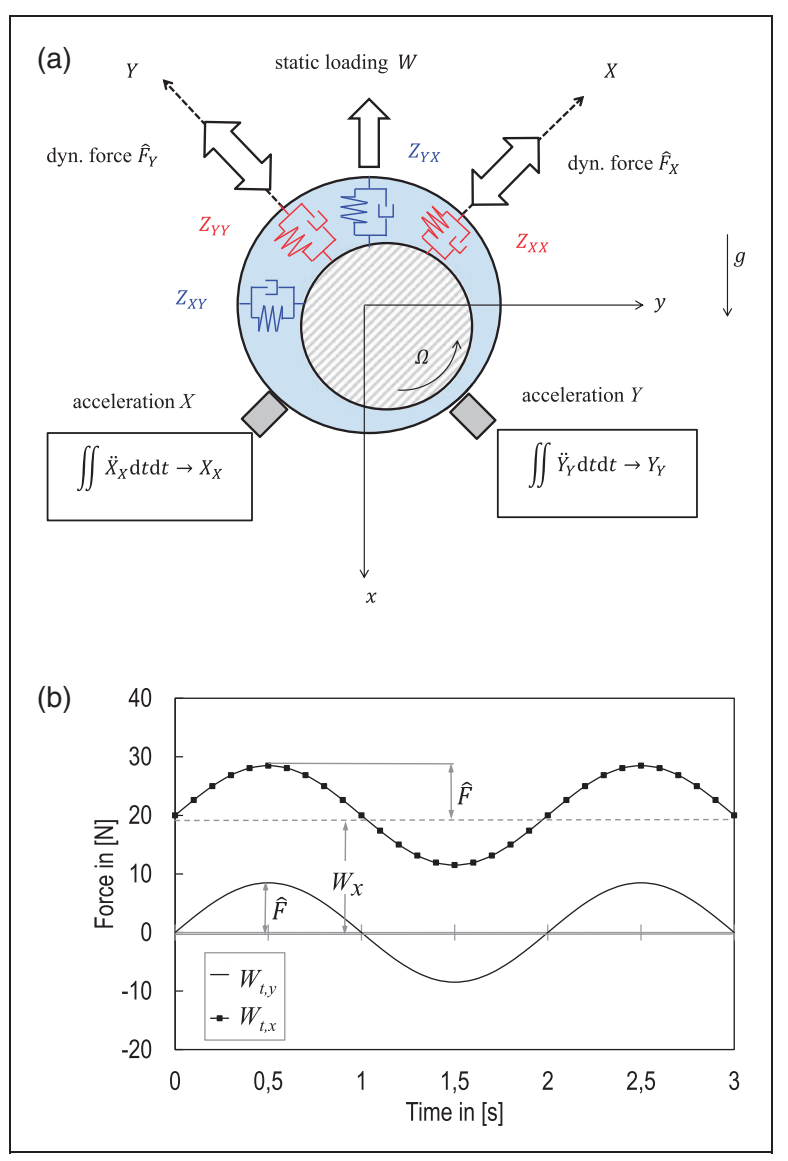

Figure 9. (a) Schematic experimental setup of a GFB parameter identification according to Rudloff et al. ${ }^{28}$ (b) Schematic total loading vs. time.

design as that given in Table 1. Several hysteresis tests by varying displacement amplitudes, static load and frequency are carried out. The dynamic structural model is validated against the test, where an optimisation identified a model stiffness of $k_{t} \approx 400 \mathrm{kN} / \mathrm{m}$. Three different loadings are considered $-20,30$ and $40 \mathrm{~N}$ - while the rotor speed is varied between 21,600 and $35,400 \mathrm{r} / \mathrm{min}$. The structural damping of the elastic structure is described by a structural loss parameter $\gamma=0.3$, which is a typical value for first generation GFBs. ${ }^{28}$ The calculated structural losses of the dynamic hysteresis model result in values of the same range. The estimation of the dynamic bump stiffness considers dynamic forces, which are based on the shaker force $\hat{F}$ in a range of $10-14 \mathrm{~N}$. Because the highest bump loading is in the same direction as the static loading $W_{x}$ (Figure 9(a)) only the mean vertical component $\hat{F}_{x}=1 / \sqrt{2} \hat{F}$ is considered. Due to the assumption of linear system behaviour, the dynamic force amplitude $\hat{F}_{p, i}$ is scaled

$$
\hat{F}_{p, i}=\frac{\hat{F}_{x}}{W_{x}} F_{p, 0, i}
$$

where $F_{p, 0, i}$ is related to the static reaction forces of the acting bump loads due to the pressure field. 
Hence, for solving the dynamic bump stiffness model a dynamic force is applied

$$
F_{p, i}(t)=F_{p, 0, i}+\hat{F}_{p, i} \cos (\omega t)
$$

The first term $F_{p, 0, i}$ is the static term. The second is the dynamic term, where the scaling ratio $\hat{F}_{x} / W_{x}$ is based on the main loading direction $x$. The experimental bump structure analysis of Larsen ${ }^{33}$ showed that the excitation frequency has no significant influence on the dynamic stiffness. The same effect can be calculated with the bump structure model presented here. Therefore, the dynamic stiffness is calculated for one discrete frequency of $1 \mathrm{~Hz}$.

In Figures 10 and 11 the stiffness values versus the excitation frequency for different rotor speeds $(21,600$ $35,400 \mathrm{r} / \mathrm{min})$ and static bearing loads $(20-40 \mathrm{~N})$ are plotted, where the experiments are shown in the sub plots (a)-(c) and the simulations in (d)-(f). The overall trend of the experiments, ${ }^{28}$ can be captured by both models (linear and non-linear structure) (Figures 10 and 11(d)-(f)). However, a trend reversal in the experimental data is shown at the frequency $f \approx 380 \mathrm{~Hz}$ due to a resonance of the shaker system. ${ }^{28}$ Furthermore, the experimental main stiffness values in the $X$ and $Y$ directions are much higher compared to the simulation. Clearance variation along the bearing surface due to manufacturing uncertainties may generate these deviations. Due to variations along the circumferential direction the converging gap is amplified, stiffens the system and generates more damping. ${ }^{23}$ The linear structural model underpredicts obviously the direct stiffness in comparison to the non-linear structural model and both models predict smaller direct stiffness in comparison to the experimental results. The linear structural model neglects the increased structural stiffness due to bump interaction, contact forces and the effect of dynamic stiffness. The underprediction of stiffness values is more distinct at higher loadings due to additional stiffening effects from the gas film and the elastic structure. Considering a simple mechanical model of two springs (gas film and structure), where both springs are connected in series, the impact of the elastic structure will be more notable if the gas film becomes very stiff. Furthermore, the linear model shows smaller differences of the cross-coupling stiffness. If the difference decreases, the stiffness matrix will become less skew-symmetric and that will result in higher threshold speeds of linear instability. Hence, the non-linear model is suitable for a robust and conservative estimation of the onset speed of instability. Figures 12 and 13 show damping parameters vs. excitation frequency. Both models show similar trends compared to the experiment, except that the cross-coupling damping in the $X Y$ direction is underpredicted. Interestingly, the damping level of the linear model is too low in the $Y$ direction and the damping of the nonlinear model shows higher values, which correlate better with the measurement results. Nevertheless, there is still a significant deviation between experimental stiffness values of main stiffness directions $(X$ and $Y$ ) and the damping is still under-predicted
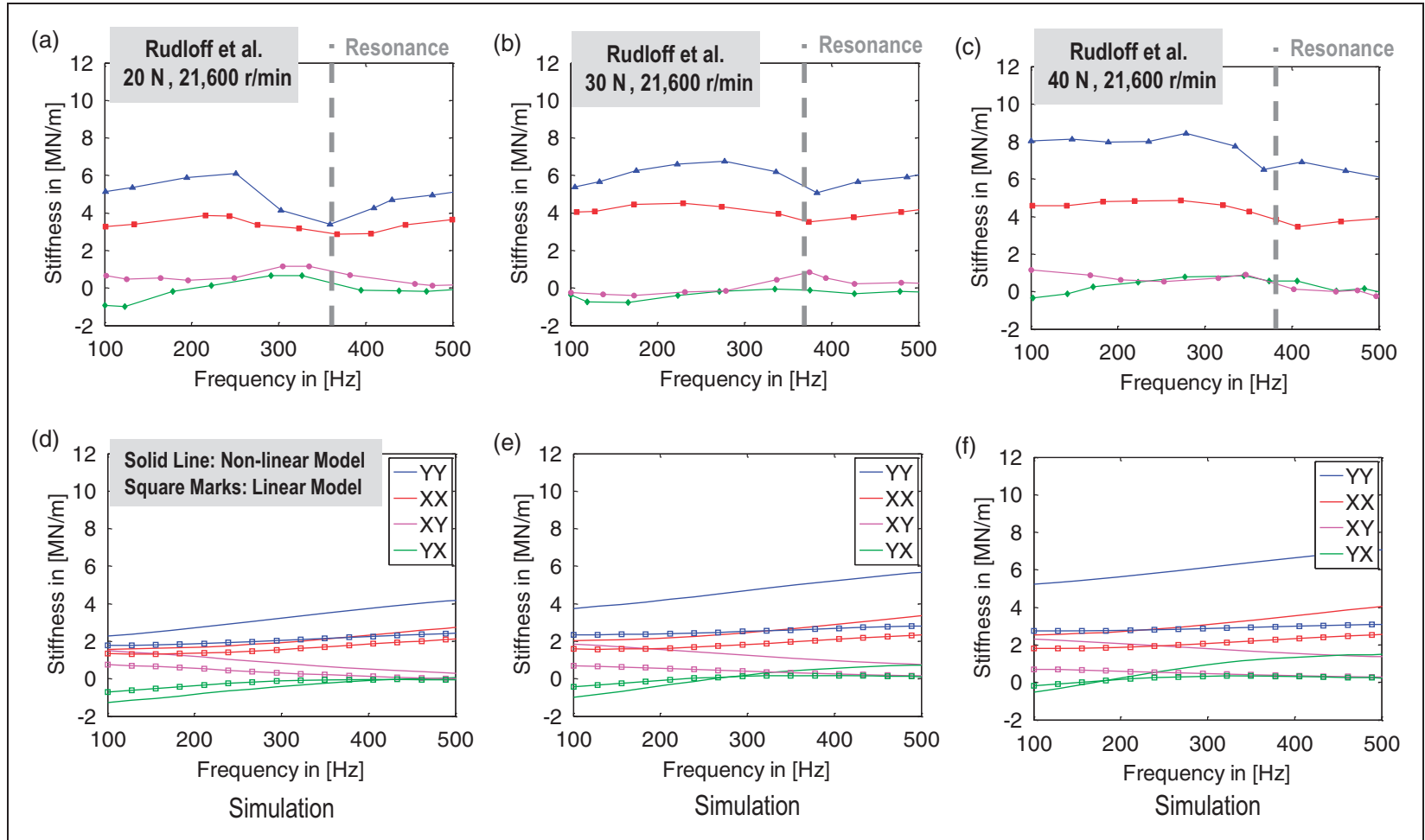

Figure 10. Stiffness parameters for $20-40 \mathrm{~N}$ and $21,600 \mathrm{r} / \mathrm{min}$. Experiments (a)-(c) vs. simulation (d)-(f). 


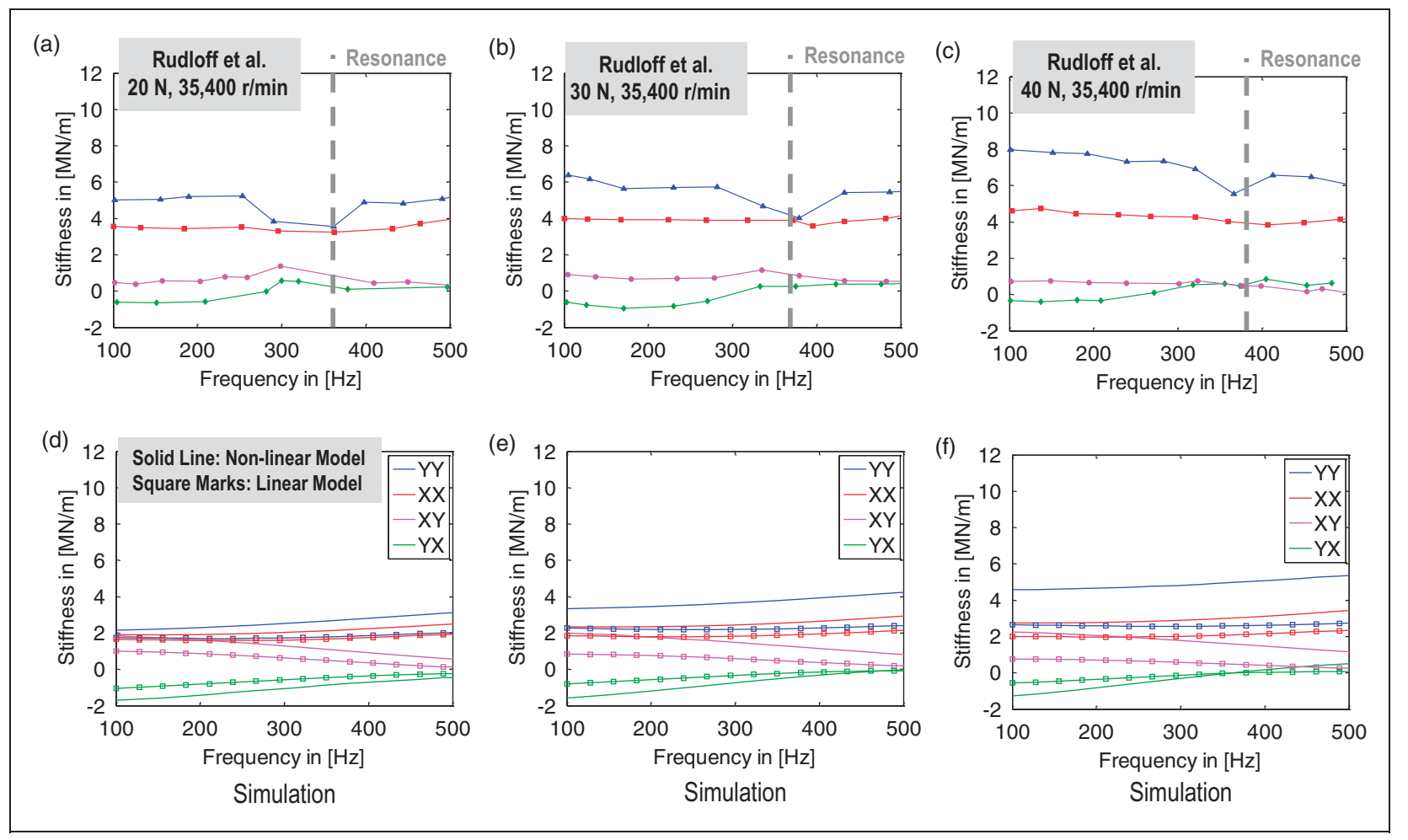

Figure II. Stiffness parameters for $20-40 \mathrm{~N}$ and 35,400 r/min. Experiments (a)-(c) vs. simulation (d)-(f).

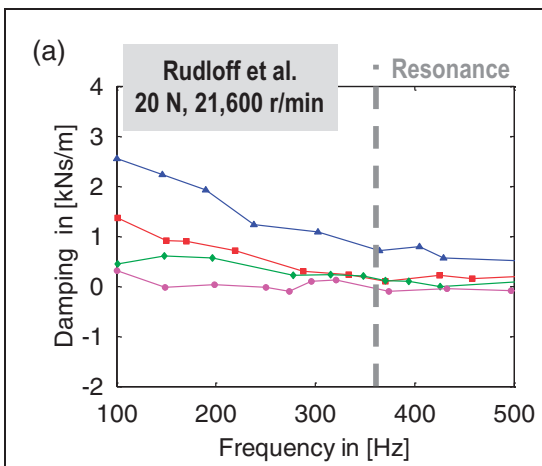

(d)

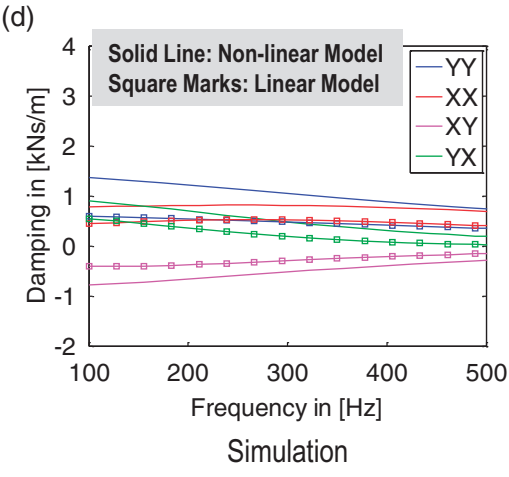

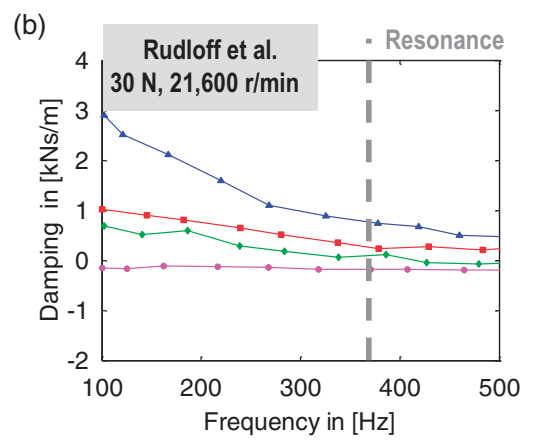

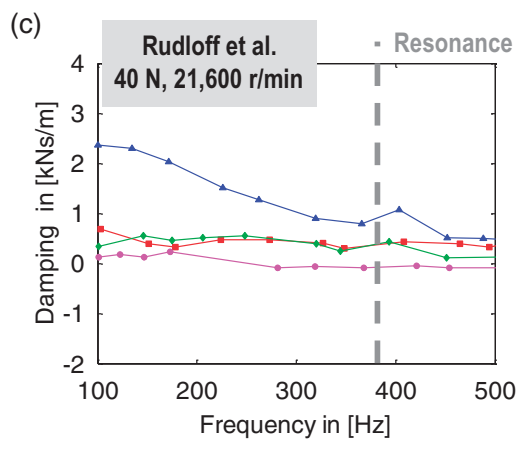

(e)

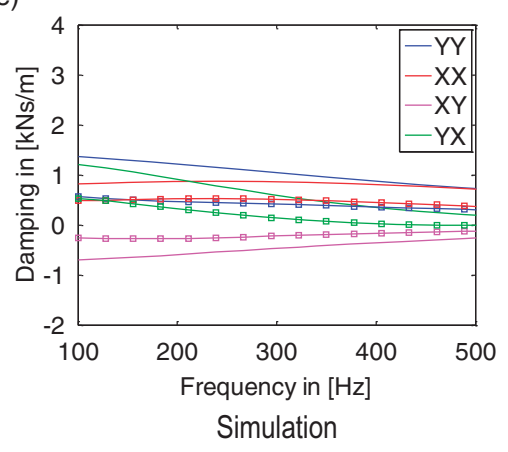

(f)

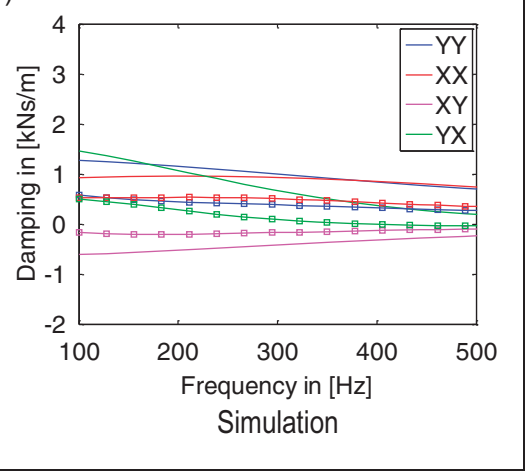

Figure 12. Damping parameters for $20-40 \mathrm{~N}$ and $21,600 \mathrm{r} / \mathrm{min}$. Experiments (a)-(c) vs. simulation (d)-(f).

for lower excitation frequency in the $Y$ direction. A sensitive analysis is applied to capture influences of:

- friction coefficients;

- bearing clearances;

- and dynamic shaker forces.
Note that the bearing clearance was changed only linearly with $\pm 20 \%$. However, due to manufacturing uncertainties the clearance can vary along circumferential and axial directions, as mentioned by Rudloff et al. ${ }^{28}$ This variation has the same effect on the bearing clearance $c$, e.g. pre-loaded bearings with shims. ${ }^{24}$ 


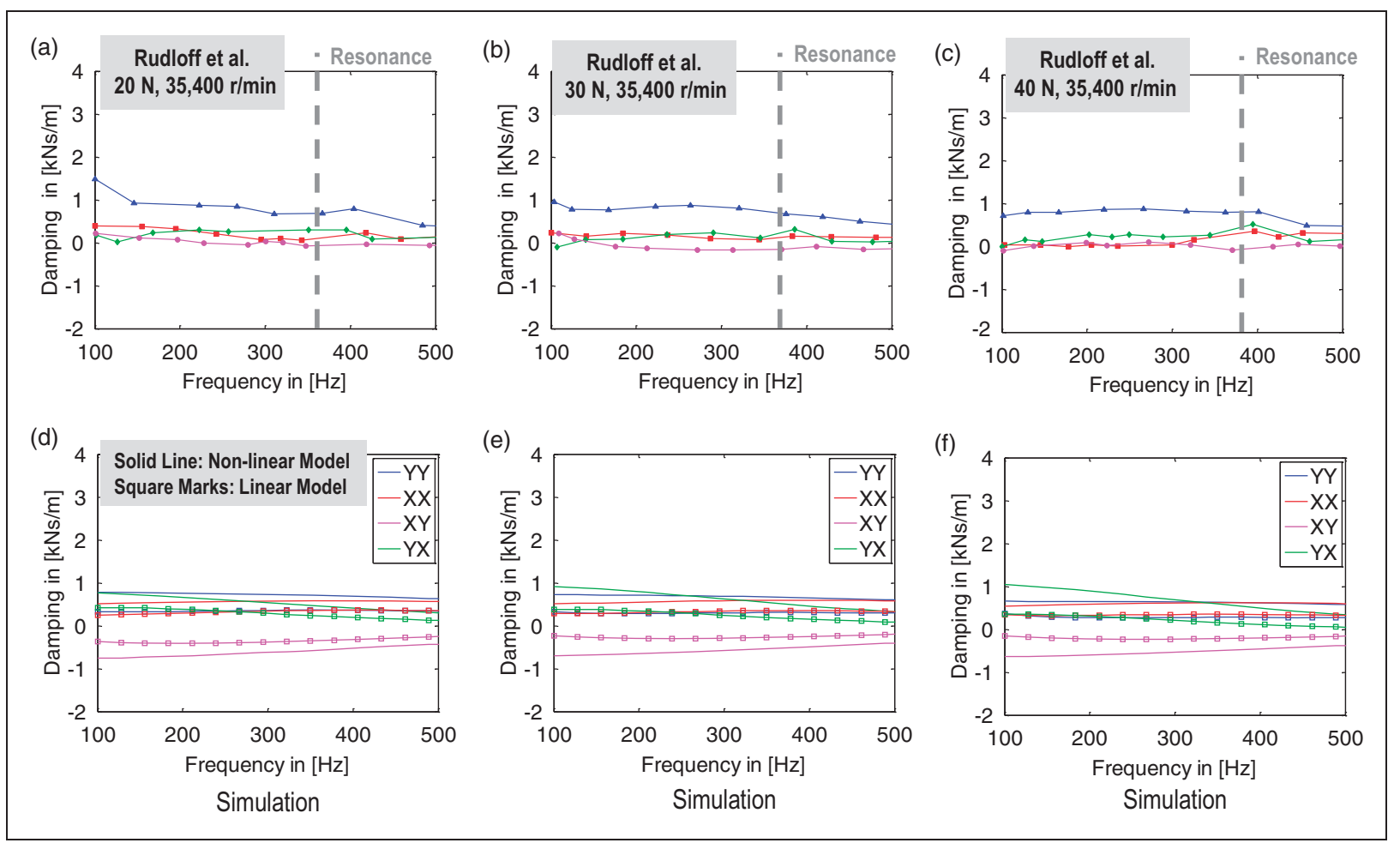

Figure 13. Damping parameters for $20-40 \mathrm{~N}$ and 35,400 r/min. Experiments (a)-(c) vs. simulation (d)-(f).

However, due to the missing information it is neglected. The last point is an adaptation to Rudloff et al.'s experiment ${ }^{28}$ and will be necessary to show the impact of higher shaker forces. For a pre-design modal analysis using equation (1) the bearing parameters can be estimated without the shaker forces. Thermal effects may influence the parameter identification, especially for higher loads and rotor speeds. However, investigations at the Berlin Institute of Technology have shown very moderate temperatures at the journal surface $T<40^{\circ} \mathrm{C}$ at $40 \mathrm{~N}$ static load at $30,000 \mathrm{r} / \mathrm{min}$ with a comparable 1.5 inch bearing. ${ }^{39} \mathrm{In}$ addition, the experiments of Rudloff et al. ${ }^{28}$ were performed at room temperature.

\section{Impact of the nominal clearance}

In this section the nominal bearing clearance is varied between $\pm 20 \%$ compared to a standard value of $c=32 \mu \mathrm{m}$, by using the non-linear structural model, as shown before. Note that the nominal radial clearance is affected by manufacturing tolerances. It is usually experimentally estimated. ${ }^{33}$ It is necessary to state the film function (equation (3)). Due to fabrication uncertainties, some changes in the nominal clearance are possible. Figure 14 shows bearing parameters under 20 and $30 \mathrm{~N}$ static loading conditions and $21,600 \mathrm{r} / \mathrm{min}$. A decreasing clearance results in higher direct stiffness and damping. In parallel, the cross-coupling stiffness differences rise. An evaluation of the clearance shows a very sensitive behaviour, which yields to high impacts on the bearing parameter estimation. This highly sensitive influence has two reasons: First, a direct impact on the film function is present, which appears in cubic terms $\left(h^{3}\right)$ in the RE. Second, narrow clearance results in lower journal displacements. Thus, the loading zone is wider, activates more bumps and, finally, generates higher dynamic stiffness beside the pressure peak maximum (indicated by arrows) (Figure 15).

In Figure 15, the static and dynamic stiffness are plotted for increasing rotor speeds (blue line: $21,600 \mathrm{r} / \mathrm{min}$ and red line: $35,400 \mathrm{r} / \mathrm{min}$ ). In general, bumps are moving rightward and start to stick at bump location 8-9. In the loading zone (bump 1022) the static stiffness is reduced significantly compared to the dynamic stiffness. The overall dynamic stiffness is raised compared to the static stiffness, because local hysteresis includes higher slopes. The maximum peak decreases for smaller clearances, higher rotor speeds and lower loadings. An increasing nominal clearance amplifies the effect of relative journal displacements and generates higher peak pressures (Appendix: Figure 21 and 22). Higher rotor speed reduces the journal centre displacement as well, while higher static loadings activate more bums, because the loading zone has increased. However, the static loading $40 \mathrm{~N}$ only generates a moderately gain of stiffness due to the relative moderate increase of loading compared to the $20 \mathrm{~N}$ case. Interestingly, the static stiffness of the higher rotor speeds and higher clearances is slightly increased for the bumps 11-18. Due to a wider zone more bumps are activated. Furthermore, the resisting friction forces generate more sticking bumps (bump 1-10). 


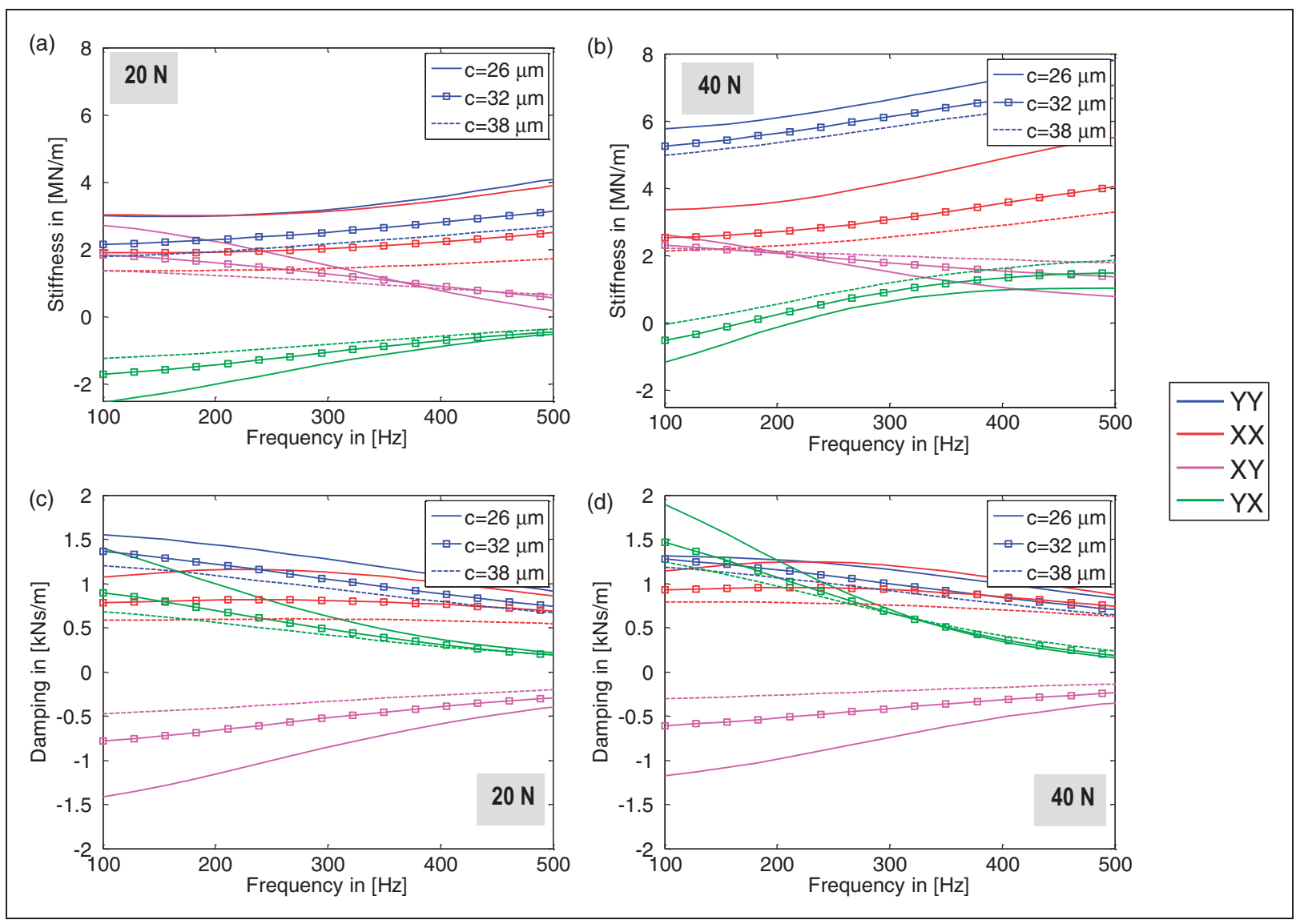

Figure 14. Impact of nominal bearing clearance: Bearing Parameters vs. excitation frequency (21,600 r/min 20 and $40 \mathrm{~N})$.

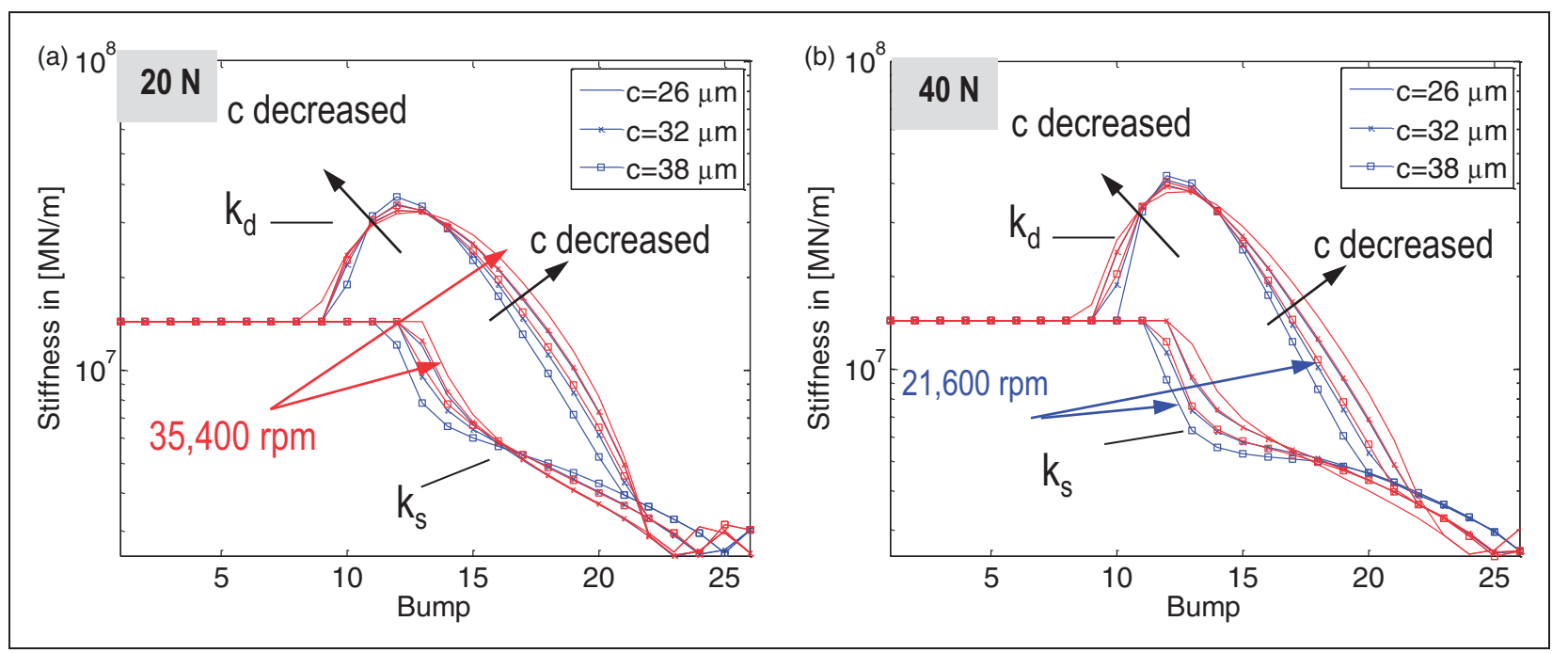

Figure 15. Impact of nominal bearing clearance: Structural stiffness, (a) $20 \mathrm{~N}$ and (b) $40 \mathrm{~N}$ at $21,600 \mathrm{r} / \mathrm{min}$ (blue) and 35,400 r/min (red).

\section{Impact of the frictional contacts inside the elastic structure}

The second sensitive analysis considers a variation of the friction coefficient $\mu$. The coefficient is increased for this study from 0.1 to 0.15 , which is still in the range of steel-steel contacts. Figure 16 shows the bearing parameters for the 20 and $40 \mathrm{~N}$ load cases at
$21,600 \mathrm{r} / \mathrm{min}$ only. For the $20 \mathrm{~N}$ loading, both stiffness and damping show no significant change in consequence of an increasing friction. However, when comparing the structural stiffness values, the same trend due to loading and rotor speed is shown as discussed in sections before. Increasing the friction coefficient results in higher stiffness (static and dynamic) and more sticking bumps, as indicated by the static 

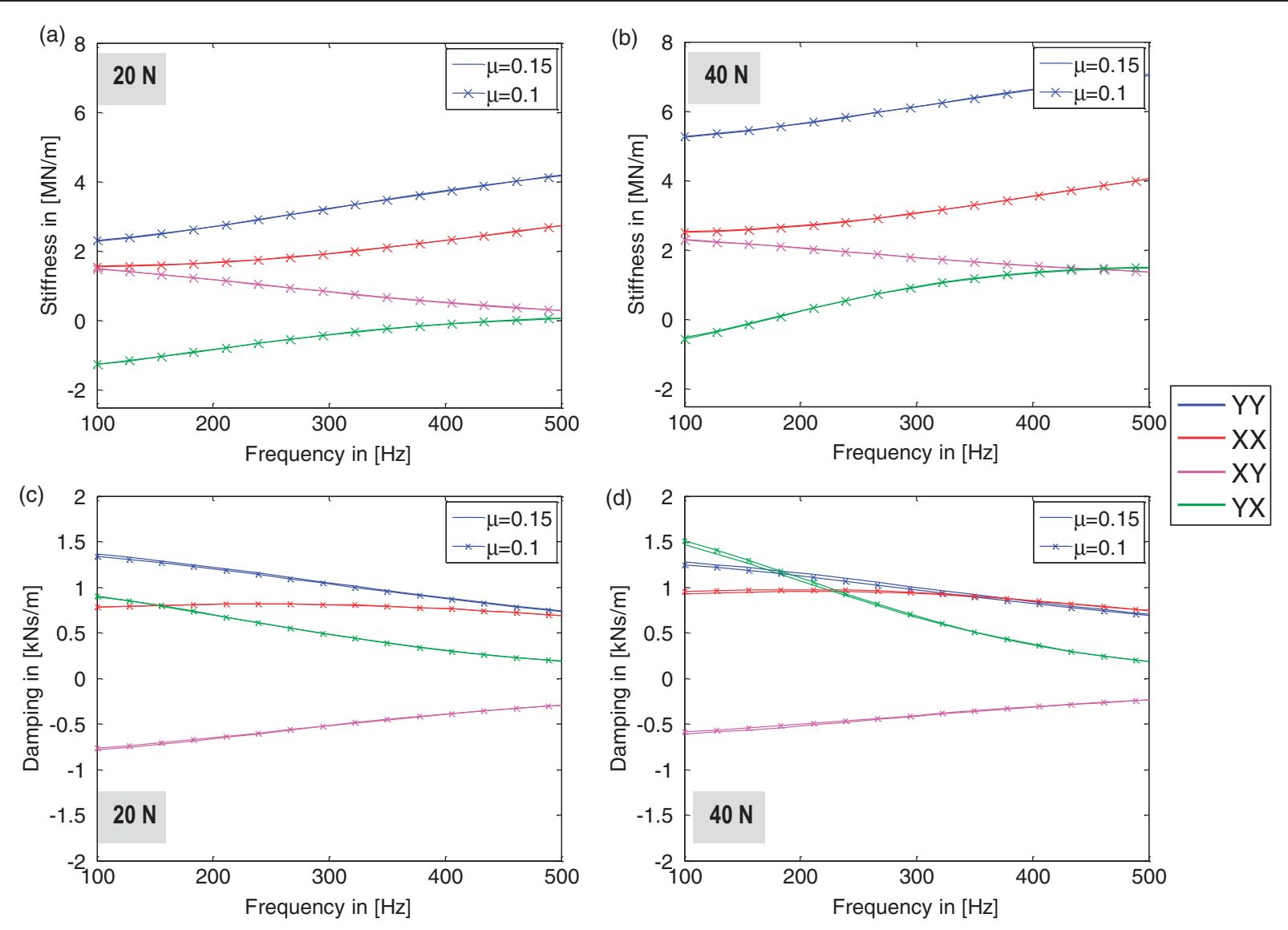

Figure 16. Impact of frictional contacts: Bearing Parameters vs. excitation frequency $(21,600 \mathrm{r} / \mathrm{min} 20$ and $40 \mathrm{~N})$.
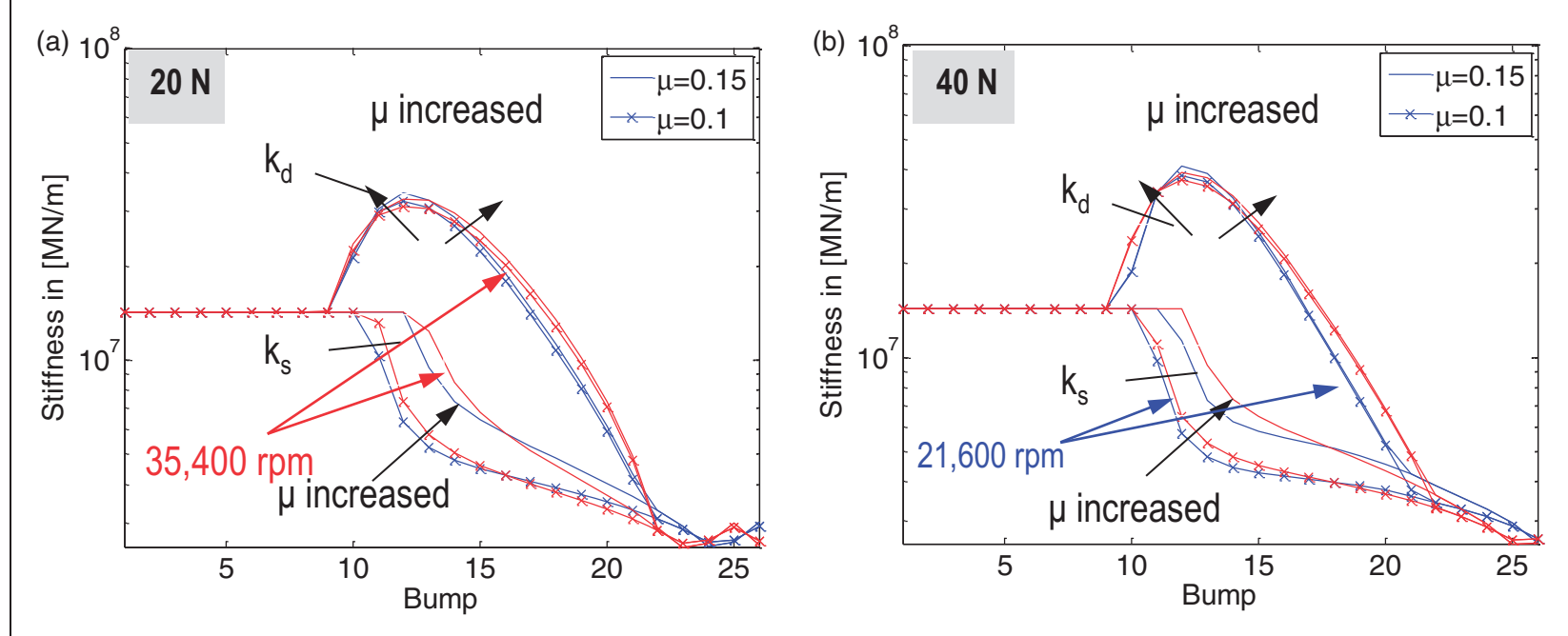

Figure 17. Impact of frictional contacts: Structural stiffness, (a) $20 \mathrm{~N}$ and (b) $40 \mathrm{~N}$ at $21,600 \mathrm{r} / \mathrm{min}$ (blue) and $35,400 \mathrm{r} / \mathrm{min}$ (red).

stiffness (constant stiffness). But higher structural stiffness has no direct impact on the fluid film function; it is only included in the compliant term $h_{c}$ of the fluid film function. Hence, the link to the pressure field and the resulting bearing parameters is less sensitive compared to the nominal clearance.
Figure 17 shows the structural stiffness, where the dynamic stiffness $k_{d}$ is increased compared to the static stiffness $k_{s}$. Higher friction coefficient slightly increases the dynamic stiffness. However, the effect is not shown in the results of the linearised bearing parameters. Comparing the influence of the compliant 
term $h_{c}$ of the film function with the effect of the nominal clearance: The compliant term $h_{c}$ is only a fraction of the overall film thickness $h(\max .4 .2 \%$ at $40 \mathrm{~N}$ and 21,500 r/min). But changes of the clearance have a direct impact on the film function $(h \sim c)$ and the bump activation. Hence, for this bearing design and load levels changes in friction has a small influence on the linearised bearing parameters.

\section{Impact of the dynamic loading in experimental parameter identification}

Figure 9(a) shows the experimental setup schematically. The parameter identification is founded on a linear time-invariant system of equation (19), where all matrix elements are transferred into the frequency domain. The impedance matrix includes the unknown bearing parameters. A successive excitation with two forces $\hat{F}$ in both directions $X$ and $Y$ by using electromagnetic shaker is applied, where cross coupling and mass inertia effects are neglected in the force matrix. The output matrix considers the displacements that can be integrated from the measured accelerations of the sensors placed in the $X$ and $Y$ direction

$$
\begin{aligned}
& {\left[\begin{array}{cc}
\hat{F}_{X}(\omega) & 0 \\
0 & \hat{F}_{Y}(\omega)
\end{array}\right]} \\
& =\left[\begin{array}{cc}
z_{X X}(\omega) & z_{X Y}(\omega) \\
z_{Y X}(\omega) & z_{Y Y}(\omega)
\end{array}\right]\left[\begin{array}{cc}
\hat{X}_{X}(\omega) & \hat{X}_{Y}(\omega) \\
\hat{Y}_{X}(\omega) & \hat{Y}_{Y}(\omega)
\end{array}\right]
\end{aligned}
$$

An inversion of the displacement matrix yields to the unknown impedances

$$
\begin{aligned}
& {\left[\begin{array}{cc}
z_{X X} & z_{X Y} \\
z_{Y X} & z_{Y Y}
\end{array}\right]} \\
& =\frac{1}{\hat{X}_{X} \hat{Y}_{Y}-\hat{X}_{Y} \hat{Y}_{X}}\left[\begin{array}{cc}
\hat{F}_{X} & 0 \\
0 & \hat{F}_{Y}
\end{array}\right]\left[\begin{array}{cc}
\hat{Y}_{Y} & -\hat{X}_{Y} \\
-\hat{Y}_{X} & \hat{X}_{X}
\end{array}\right]
\end{aligned}
$$

Experimental analysis on a comparable 1.5 inch gas foil bearing have shown, that $\hat{X}_{Y} \hat{Y}_{X} \ll \hat{X}_{X} \hat{Y}_{Y}{ }^{39}$ Hence, the product of cross coupling displacements $\hat{X}_{Y} \hat{Y}_{X}$ is neglected

$$
\begin{aligned}
& {\left[\begin{array}{cc}
z_{X X} & z_{X Y} \\
z_{Y X} & z_{Y Y}
\end{array}\right] \approx\left[\begin{array}{cc}
\hat{F}_{X} & \hat{F}_{X} \hat{X}_{Y} \\
\hat{X}_{X} & \hat{X}_{X} \hat{Y}_{Y} \\
\hat{F}_{Y} \hat{Y}_{X} & \hat{F}_{Y} \\
\hat{\hat{X}}_{X} \hat{Y}_{Y} & \frac{\hat{Y}_{Y}}{\hat{Y}_{Y}}
\end{array}\right]} \\
& =\underbrace{\hat{F}_{X}\left[\begin{array}{cc}
\frac{1}{\hat{X}_{X}} & -\frac{\hat{X}_{Y}}{\hat{X}_{X} \hat{Y}_{Y}} \\
0 & 0
\end{array}\right]}_{A}+\underbrace{\hat{F}_{Y}\left[\begin{array}{cc}
0 & 0 \\
-\frac{\hat{Y}_{X}}{\hat{X}_{X} \hat{Y}_{Y}} & \frac{1}{\hat{Y}_{Y}}
\end{array}\right]}_{B}
\end{aligned}
$$

Equation (21)'s impedance matrix is the sum of two matrices A and B. They include linearly independent excitations (shaker forces $\hat{F}_{X}$ and $\hat{F}_{Y \text { ) }}$ in $X-$ and
$Y$-direction. Each direction generates one column of the impedance matrix. In fact, static bearing loadings (vertical $x$-direction), which are implicitly included in the measured displacements, are superimposed with the dynamic loadings of excitation (10$14 \mathrm{~N}$, Rudloff et al. ${ }^{28}$ ) Furthermore, cross-coupling effects of the two independent excitations are only present in the cross-coupling direction (matrix elements $i j$ and $j i)$ due to the assumption of $\hat{X}_{Y} \hat{Y}_{X} \ll \hat{X}_{X} \hat{Y}_{Y}$. A comparison of the experimental linearised bearing parameter towards numerical results based on an infinitely small perturbation is shown in Figures 10-13. The results show a significant deviation between simulations and experiments. In fact, Lunds approach suits well for displacements in a range up to $0.4 c$. The maximal displacement range of Rudloff et al. ${ }^{28}$ is approximately $7 \mu \mathrm{m}$, which is approx. $21 \%$ of the nominal clearance $(c=32 \mu \mathrm{m})$. However, the validity of Lunds method is based on rigid bearings, without a compliant structure. As shown, the elastic structure is mainly affected by bearing loadings and rotor speeds, as shown in Figures 10-13. Hence, a rough adaption for infinitely small parameter identification is introduced by using total bearing loadings, especially if the dynamic force magnitude is significant.

Considering a non-linear progressive system, as schematically shown in Figure 18, two cases can be discussed: If an infinitely small perturbation is applied around the equilibrium position $\left(W_{x}, x\right)$ the dynamic force $\hat{F}$ becomes zero. Due to a quasi-linear characteristic a linearisation based on infinitely small perturbations can be applied. However, if the dynamic perturbation force $\hat{F}$ is increased, a higher displacement $x^{\prime}$ with the total force $W_{t}$ is present. Thus, the gradient, which correlate with the bearing parameters, of the total state is steeper compared to the infinitely small perturbation point $\left(k^{\prime}>k\right)$. For better approximation an extension of the Taylor series with higher orders $\geqslant \mathcal{O}\left(\Delta^{2}\right)$ needs no be applied to equation (5). However, it is assumed that the loading zone of the gas foil bearing mainly generates the bearing impedance due to the highest number of active bumps. The loading zone is significantly influenced by total loadings, where the maximum peak of total loadings in each direction is applied (Figure 9(b)). Hence, the total state of Figure 18 can be separately evaluated for each direction. Around the total states, the gradients $k^{\prime}$ can be approximated by applying infinitely small perturbations. Therefore, the total loads (static and dynamic) due to shakers are given by equation (22). These loads are used to estimate the pressure field of the zero order RE

$$
W_{t, X}=\left\{W_{x}+\frac{1}{\sqrt{2}} \hat{F}_{X},-\frac{1}{\sqrt{2}} \hat{F}_{X}\right\}^{\mathrm{T}}
$$

and

$W_{t, Y}=\left\{W_{x}+\frac{1}{\sqrt{2}} \hat{F}_{X},+\frac{1}{\sqrt{2}} \hat{F}_{X}\right\}^{\mathrm{T}}$ 
Indeed the matrix elements of equation (21) are independent in each direction. Thus, only the excitation in one direction will allow a bearing parameter calculation of the considered direction. Therefore, the matrix rows are separated into two matrices equation (21). Applying the local loadings of equation (22) for two different calculations ( $X$ and $Y$ excitation) will result in two impedance matrices ( $X$ and $Y$ excitation). A comparison of the calculated matrices with equation (21) will deliver a final impedance matrix based on total loads due to two excitations. The first row of

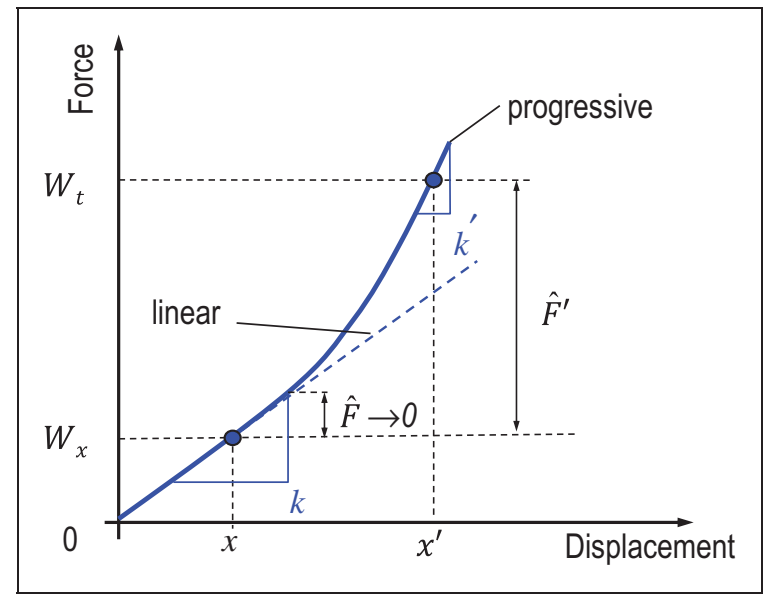

Figure 18. Schematic force displacement relationship of a non-linear progressive system. the $X$ impedance matrix will deliver the bearing parameters in $X$ direction. In addition, the other parameters of the calculated $X$ direction matrix are set to zero (equation (21)). The calculation for the $Y$ direction is based on the same assumption. It will deliver the second row of the impedance matrix, where the other elements ( $X$-direction) of this calculation are set to zero, as well. A superposition of these two different matrices (A and B) finally results in the overall impedance matrix.

Figures 19 and 20 show the bearing parameters of the simulation with the total bearing loadings due to shaker forces and a rotor speeds of 21,600 and $27,600 \mathrm{r} / \mathrm{min}$. The level of the direct stiffness is increased and shows a good agreement towards the measurements for higher excitation frequency. For lower frequencies the direct values in $x$ and $y$ direction are underpredicted. However, the behaviour of the cross coupling effect is well correlated to measurements. The damping values still show some deviations. In main direction the damping values are slightly smaller for lower excitation frequencies. In addition, the cross coupling damping $X Y$ delivers values that are too small. Due to the sensitivity of the bearing clearance in comparison a variation of the clearance due to manufacturing uncertainties may generate the deviations for stiffness and damping at lower frequencies. Nevertheless, the adaptation of shaker forces improves the bearing parameter correlation especially for stiffness values.
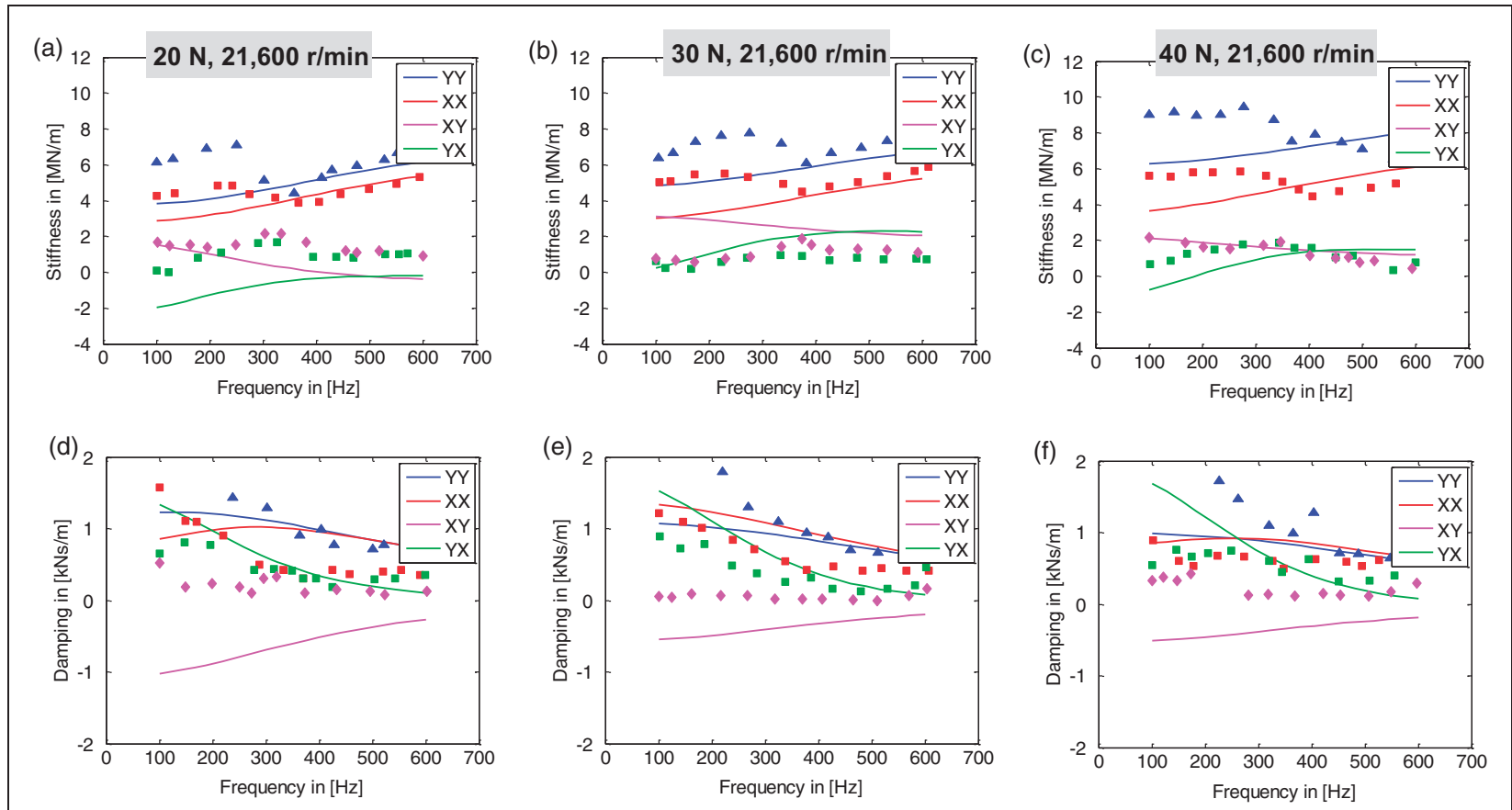

Figure 19. Bearing parameters based on experimental setup adaption, solid line: Simulations $(20-40 \mathrm{~N}, 21,600 \mathrm{r} / \mathrm{min})$, $\mathrm{marks}$ : Experimental results from Rudloff et al. ${ }^{28}$ 


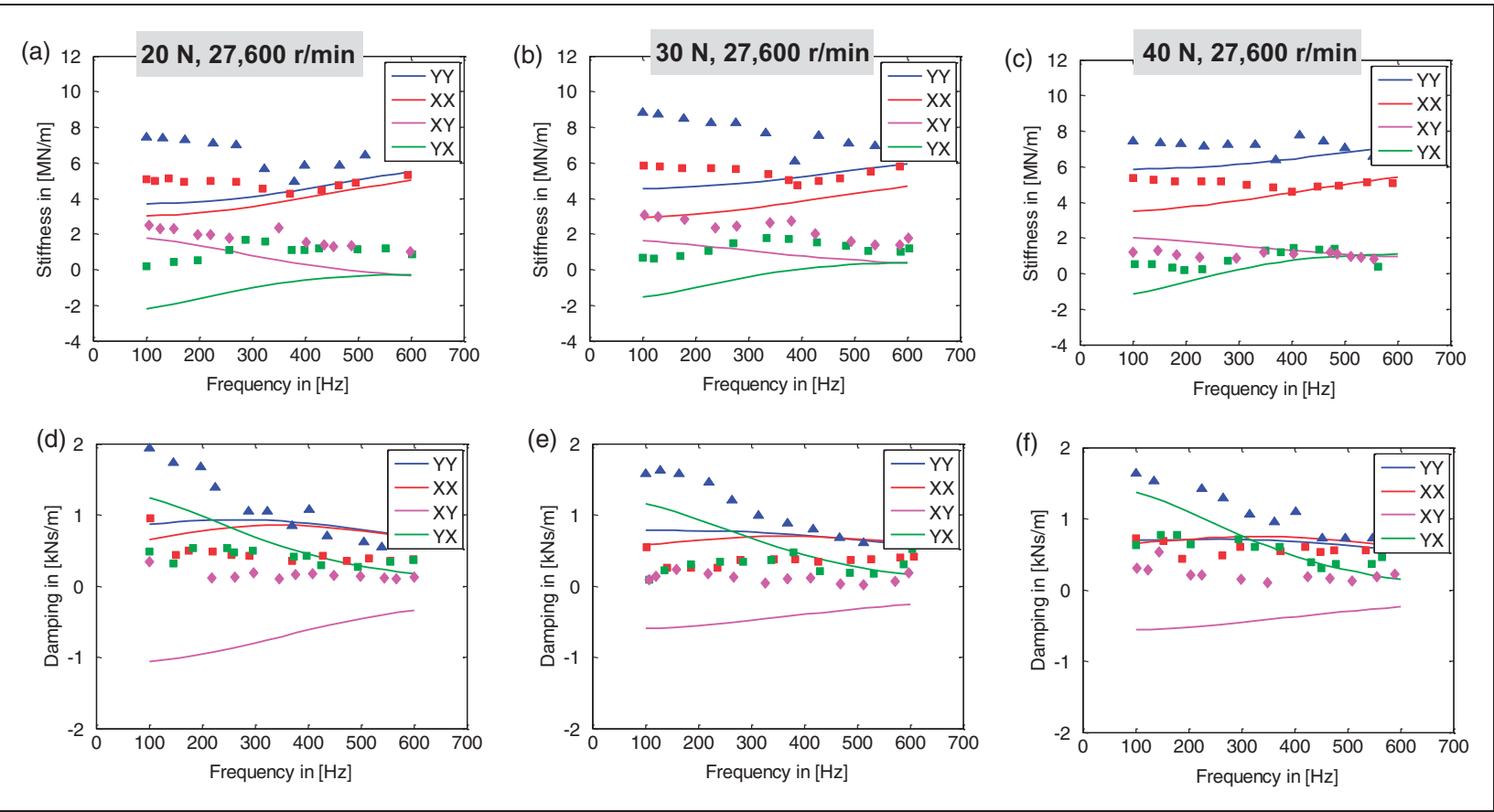

Figure 20. Bearing parameters based on experimental setup adaption, solid line: Simulations (20-40 N, 27,600 r/min), marks: Experimental results from Rudloff et al. ${ }^{28}$.

\section{Conclusion}

This paper has used a perturbation method related to Lund's work. ${ }^{10}$ The model couples the fluid film simulation with a non-linear structural model including frictional contacts and bump interactions. A method has been applied to calculate a dynamic structural stiffness ${ }^{19}$ The dynamic stiffness model is validated against experimental data of Larsen et al. ${ }^{31}$ In the second part of the paper the linearised model is validated by using data from Rudloff et al. ${ }^{28}$ The results of this paper show that:

- dynamic forces may have a significant impact on the bearing parameter identification;

- a linear model underpredicts the stiffness and damping values compared to a non-linear model including static and dynamic stiffness;

- friction has a significant impact on the elastic structure. But the influence on bearing parameters of relatively small static loads $<40 \mathrm{~N}$ is insignificant due to a non-sensitive impact of the compliant part of the film function;

- the nominal clearance $c$ has a direct link to the pressure field and has a significant influence on the bearing parameter;

- damping shows some deviations and is underpredicted for lower frequencies.

As shown in the results (Figures 19 and 20) dynamic forces may significantly affect the bearing parameter identification. For the evaluation of critical speeds and threshold speeds by using equation (1), when considering a well-balanced system, the infinitely small perturbation based on the non-linear structural models will result in the most realistic values. To deliver more detailed experimental data for a bearing parameters identification based on small perturbations, a test rig at Berlin Institute of Technology is used for further investigation including preloaded and higher loaded bearings.

\section{Declaration of Conflicting Interests}

The author(s) declared no potential conflicts of interest with respect to the research, authorship, and/or publication of this article.

\section{Funding}

The author(s) received no financial support for the research, authorship, and/or publication of this article.

\section{References}

1. Hoffmann R, Pronobis T and Liebich R. Non-linear stability analysis of a modified gas foil bearing structure. In: Proceedings of the 9th IFToMM international conference on rotor dynamics, Milano, 2014. Springer.

2. Choe BS, Kim TH, Kim CH, et al. Rotordynamic behavior of $225 \mathrm{kw}(300 \mathrm{hp})$ class pms motor-generator system supported by gas foil bearings. J Eng Gas Turbines Power 2015; 137(9): 092505.

3. Heshmat H, Shapiro W and Gray S. Development of foil journal bearings for high load capacity and high speed whirl stability. J Lubr Technol 1982; 104(2): 149-156.

4. Heshmat H. Advancements in the performance of aerodynamic foil journal bearings: high speed and load capability. J Tribol 1994; 116(2): 287-294. 
5. Sim K, Lee YB, Kim TH, et al. Rotordynamic performance of shimmed gas foil bearings for oil-free turbochargers. J Tribol 2012; 134(3): 031102-1031102-11.

6. San Andrés L and Kim TH. Forced nonlinear response of gas foil bearing supported rotors. Tribol Int 2008; 41(8): 704-715.

7. Balducchi F, Arghir M and Gaudillere S. Experimental analysis of the unbalance response of rigid rotors supported on aerodynamic foil bearings. In: ASME Turbo Expo 2014: Turbine Technical Conference and Exposition, Paper No. GT2014-25552, Düsseldorf, Germany, 16-20 June 2014, p.V07BT32A009; 12pp.

8. Nguyen-Schäfer H. Rotordynamics of automotive turbochargers: Linear and nonlinear rotordynamics-Bearing design-rotor balancing. Springer, 2012.

9. Thomsen JJ. Vibrations and stability: Advanced theory, analysis, and tools. Berlin Heidelberg: Springer, 2003.

10. Lund JW. Calculation of stiffness and damping properties of gas bearings. $J$ Lubr Technol 1968; 90(4): 793-803.

11. Kim TH and San Andrés L. Heavily loaded gas foil bearings: A model anchored to test data. J Eng Gas Turbines Power 2008; 130(1): 012504-1-012504-8.

12. Kim TH and San Andrés L. Effect of side feed pressurization on the dynamic performance of gas foil bearings: A model anchored to test data. J Eng Gas Turbines Power 2009; 131(1): 012501.

13. Schiffmann J and Spakovszky ZS. Foil bearing design guidelines for improved stability. J Tribol 2013; 135: 011103-1-011103-11.

14. Kim TH and San Andrés L. Effect of mechanical preloads on the dynamic performance of gas foil bearings. In: Proceedings of the ASME STLE international joint tribology conference, 2008.

15. Kim D. Parametric studies on static and dynamic performance of air foil bearings with different top foil geometries and bump stiffness distributions. J Tribol 2007; 129(2): 354-364.

16. San Andrés L and Kim TH. Improvements to the analysis of gas foil bearings: Integration of top foil $1 \mathrm{D}$ and 2D structural models. In: Proceedings of ASME Turbo Expo 2007: Power for Land, Sea, and Air, Montreal, Canada, 14-17 May 2007, pp.779-789.

17. Hoffmann R, Pronobis $T$, and Liebich $R$. The impact of modified corrugated bump structures on the rotor dynamic performance of gas foil bearings. In: Proceedings ASME Paper No. GT2014-25636, 2014a.

18. Hoffmann R, Pronobis T, and Liebich R. Stability analysis of a pressurized gas foil bearings for high speed applications. In: Proceedings 11th international conference on turbocharging, 2014b.

19. Larsen JS, Hansen AJT and Santos IF. Experimental and theoretical analysis of a rigid rotor supported by air foil bearings. Mech Ind 2015; 16(1): 106.

20. Lee YB, Park DJ, Kim CH, et al. Operating characteristics of the bump foil journal bearings with top foil bending phenomenon and correlation among bump foils. Tribol Int 2008; 41(4): 221-233.

21. Feng K and Kaneko S. Analytical model of bump-type foil bearings using a link-spring structure and a finiteelement shell model. J Tribol 2010; 132(1): 021706-1021706-11.
22. Le Lez S, Arghir M and Frene J. A new bump-type foil bearing structure analytical model. J Eng Gas Turbines Power 2007; 129: 1047-1057.

23. Hoffmann R, Pronobis T and Liebich R. A performance analysis of a gas foil bearing including structural modifications by applying metal shims. In: Proceedings internationale tagung schwingungen in rotierenden maschinen (SIRM), 2015.

24. Carpino $\mathrm{M}$ and Talmage G. Prediction of rotor dynamic coefficients in gas lubricated foil journal bearings with corrugated sub-foils. Tribol Trans 2006; 49(3): 400-409.

25. Peng JP and Carpino M. Coulomb friction damping effects in elastically supported gas foil bearings. Tribol Trans 1994; 37(1): 918.

26. San Andrés L and Chirathadam TA. Metal mesh foil bearing: Effect of motion amplitude, rotor speed, static load, and excitation frequency on force coefficients. J Eng Gas Turbines Power 2011; 133(12): 122503.

27. Sim K, Lee YB, Song JW, et al. Identification of the dynamic performance of a gas foil journal bearing operating at high temperatures. J Mech Sci Technol 2014; 28(1): 43-51.

28. Rudloff L, Arghir M, Bonneau O, et al. Experimental analyses of a first generation foil bearing: Startup torque and dynamic coeficients. J Eng Gas Turbines Power 2011; 133(9): 092501-1-092501-9.

29. Howard S, Dellacorte C, Valco MJ, et al. Dynamic stiffness and damping characteristics of a high-temperature air foil journal bearing. Tribol Trans 2001; 44(4): 657-663.

30. Norsworthy JD. Measurement of drag torque, lift off speed, and identification of frequency dependent stiffness and damping coefficients of a shimmed bump-type foil bearing. Masters Thesis, Texas A\&M University, 2014.

31. Larsen SJ, Varela AC and Santos IF. Numerical and experimental investigation of bump foil mechanical behaviour. Tribol Int 2014; 74: 46-56.

32. Al-Bender F and Swevers J. Characterization of friction force dynamics. Control Syst, IEEE 2008; 28(6): 64-81.

33. Larsen JS. Nonlinear analysis of rotors Supported by air foil journal bearings - theory and experiments. $\mathrm{PhD}$ thesis, Technical University of Denmark, 2015.

34. Petrov EP and Ewins DJ. Generic friction models for time-domain vibration analysis of bladed discs. $J$ Turbomach 2003; 126: 184-192.

35. Le Lez S, Arghir M and Frene J. A dynamic model for dissipative structures used in bump-type foil bearings. Tribol Trans 2009; 52(1): 36-46.

36. Munz O. Implementierung eines dynamischen Reibungsmodells in eine nichtlineare GasfolienlagerStruktur. Masters Thesis, Technische Universität Berlin, 2015.

37. Qiu ZL and Tieu AK. The effect of perturbation amplitudes on eight force coefficients of journal bearings. Tribol Trans 1996; 39(2): 469-475.

38. Lund JW. Review of the concept of dynamic coefficients for fluid film journal bearings. J Tribol 1987; 109(1): 37-41.

39. Deghela U. Experimentelle Paramteridentifikation eines Gasfolienlagers unter dem Einfluss von Drehzahl und statischer Lagerlast. Master Thesis, Technische Universität Berlin, 2016. 


\section{Appendix I}

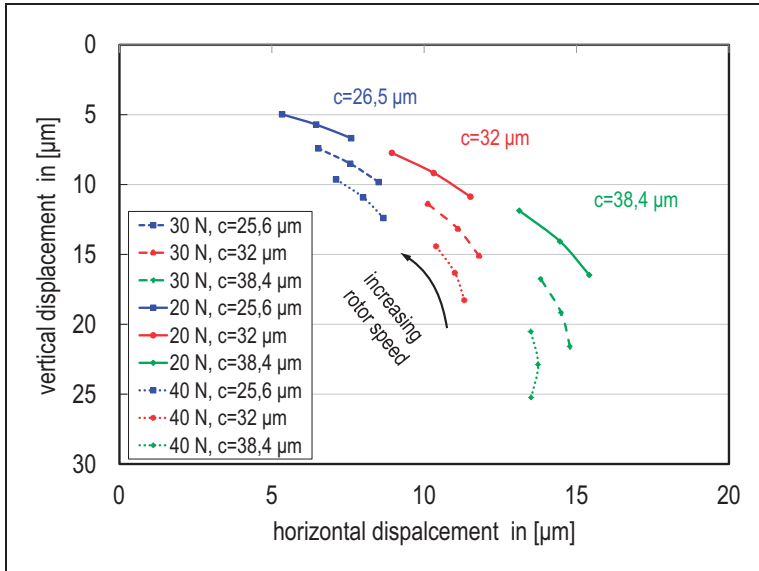

Figure 21. Impact of nominal bearing clearance on the locus curve.

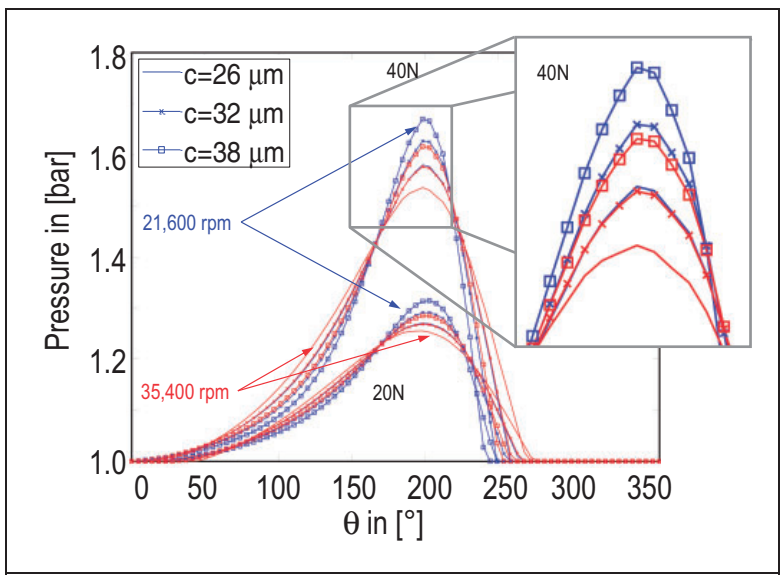

Figure 22. Impact of nominal bearing clearance on the pressure field for $40 \mathrm{~N}$ and $20 \mathrm{~N}$ and different speeds (red line: $35,400 \mathrm{r} / \mathrm{min}$ and blue line: $21,600 \mathrm{r} / \mathrm{min}$ ).

\section{Appendix 2}

\section{Notation}

A

C

c

$c_{i j}$

$c_{t}$

$E$

$e_{i}$

$\Delta e_{i}$

F

$f$ system matrix dynamic link-springmodel

damping matrix

radial bearing clearance

linearised damping value $i, j=x, y$

Petrov-model parameter

Young-modulus

journal displacement $i=x, y$

perturbed journal displacement $i=x, y$

force

dynamic force

frequency
$F_{b, x}$

$F_{p}$

$F_{S}$

$F_{x}$

$\mathbf{f}_{B}$

$\mathbf{f}_{f}$

$\mathbf{f}_{p}$

G

$h$

$\Delta h$

$\Delta \hat{h}$

$h_{b}$

$h_{c}$

$h_{c, i}$

$h_{i}$

$h_{r}$

j

$\mathbf{K}_{\text {Bump }}$

$k_{d}$

$k_{i j}$

$k_{s}$

$k_{t}$

$k_{1}$

$k_{2}$

$l$

$l_{b}$

$\Delta L$

M

$N$

$N_{b}$

$p$

$p_{a}$

$p_{i}$

$R$

$s_{b}$

$T$

$t$

$t_{b}$

$t_{f}$

$U$

u

w

$W_{i}$

$W_{t, i}$

$X$

$x$

$x_{s}$

$Y$

y

Z

$z$

$z_{i j}$ horizontal beam lever force

bump load

interaction force

horizontal reaction force

reacting force vector

friction force vector

pressure force vector

gyroscopic matrix

film thickness

vertical displacement link-spring-model

dynamic vertical displacement link-

spring-model

bump hight

compliant term of the film thickness

perturbed compliant film thickness

term $i=x, y$

perturbed film thickness $i=x, y$

rigid term of the film thickness

complex number $\mathrm{j}=\sqrt{-1}$

stiffness matrix

bump stiffness matrix

dynamic structural stiffness

linearised stiffness value $i, j=x, y$

static structural stiffness

Petrov-model stiffness parameter

interaction spring stiffness link-spring-

model

spring stiffness link-spring-model

bearing length

half bump length

horizontal displacement link-spring-

model

mass matrix

normal contact force

bump number

pressure

ambient pressure

perturbed pressure $i=x, y$

bearing / journal radius

bump pitch

temperature

time

bump thickness

top foil thickness

journal rotation speed $U=R \Omega$

displacement vector

loading vector $\mathbf{w}=\left\{W_{x}, W_{y}\right\}^{T}$

bearing load $i=x, y$

total loading $i=X, Y$

Cartesian coordinate

Cartesian coordinate

displacement Petrov-model

Cartesian coordinate

Cartesian coordinate

complex impedance matrix

Cartesian coordinate

complex impedance

$z_{i j}=k_{i j}+\mathrm{j} \omega c_{i j} i, j=x, y$ 
$\alpha \quad$ bump angle link-spring-model

$\beta \quad$ attitude angle

$\gamma \quad$ structural loss factor $\gamma=c \omega / k_{d}$

$\delta_{i} \quad$ damping ratio $i=1,2 \ldots n$

$\lambda_{i} \quad$ eigenvalue $\lambda_{i}=\delta_{i} \pm \mathrm{j} \omega_{i}^{*} i=1,2 \ldots n$

$\mu_{l} \quad$ dynamic viscosity

$\mu \quad$ friction coefficient

$v \quad$ Poisson ratio

$\omega \quad$ excitation frequency

$\Omega \quad$ rotor speed

$\Omega_{0} \quad$ onset speed of sub-synchronous

vibrations

$\theta$ $\theta_{0} \quad$ bump angle

\section{Subscripts}

$(\ldots)_{b o t} \quad$ bottom link-spring-model

$i \quad$ bump counter

$(\ldots)_{l} \quad$ left link-spring-model

$(\ldots)_{r} \quad$ right link-spring-model

$(\ldots)_{u p}$ up link-spring-model

$(\ldots)_{0} \quad$ zero order terms

(...)

(...) amplitude of a dynamic value

Time derivative 